\title{
Competing Currencies in the Laboratory
}

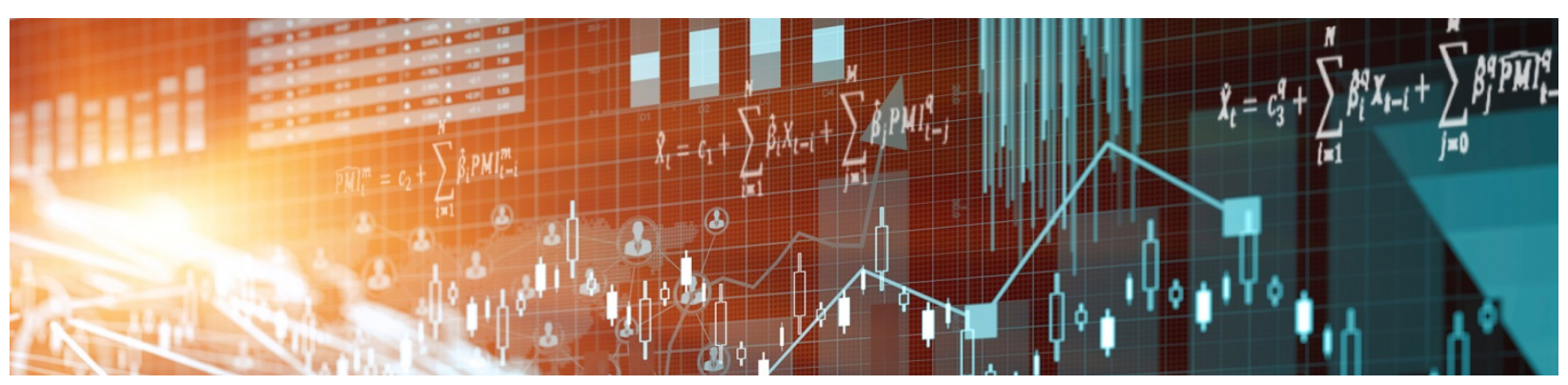

by Janet Hua Jiang and Cathy Zhang 
Bank of Canada Staff Working Paper 2017-53

December 2017

\title{
Competing Currencies in the Laboratory
}

\author{
by \\ Janet Hua Jiang ${ }^{1}$ and Cathy Zhang ${ }^{2}$ \\ ${ }^{1}$ Funds Management and Banking Department \\ Bank of Canada \\ Ottawa, Ontario, Canada K1A 0G9 \\ iiiang@bankofcanada.ca \\ 2 Department of Economics, Krannert School of Management \\ Purdue University \\ West Lafayette, Indiana, USA 47907 \\ cmzhang@purdue.edu
}




\section{Acknowledgements}

We are grateful to Tim Cason, John Duffy, Daniela Puzzello, Guillaume Rocheteau, Julian Romero, Yaroslav Rosokha, Randy Wright, and Yu Zhu for helpful discussions and comments. We also thank conference and seminar participants at the 2016 ESA North American Meetings in Tucson, 2015 ESA World Meetings in Sydney, Purdue University, and the Bank of Canada for feedback. Chineze Christopher and Stanton Hudja provided excellent research assistance. 


\begin{abstract}
We investigate competition between two intrinsically worthless currencies as a result of decentralized interactions between human subjects. We design a laboratory experiment based on a simple two-country, two-currency search model to study factors that affect circulation patterns and equilibrium selection. Experimental results indicate foreign currency acceptance rates decrease with relative country size but are not significantly affected by the degree of integration. The laboratory economies tend to converge to a unified currency regime where both currencies circulate at home and abroad, even if other regimes are theoretical possibilities. Introducing government transaction policies biased towards domestic currency significantly reduces the acceptability of foreign currency. These findings suggest government policies can serve as a coordination device when multiple currencies are available.
\end{abstract}

Bank topics: Central bank research; Digital currencies

JEL codes: C92, D83, E40

\title{
Résumé
}

Nous étudions la concurrence que se livrent deux monnaies sans valeur intrinsèque, du fait d'interactions décentralisées entre sujets humains. Nous faisons une expérience en laboratoire basée sur un modèle de prospection simple intégrant deux pays et deux monnaies. Notre but est d'étudier les facteurs ayant une incidence sur les profils de circulation et le choix de l'équilibre. D’après les résultats de l'expérience, le taux d'acceptation d'une monnaie étrangère dans un pays diminue à mesure que la taille relative de celui-ci augmente, mais il ne varie pas sensiblement selon le degré d'intégration économique. Dans les simulations en laboratoire, deux économies ont tendance à converger vers un régime monétaire unifié au sein duquel les deux monnaies circulent de part et d'autre, même si d'autres régimes sont possibles en théorie. La mise en place de politiques privilégiant l'utilisation de la monnaie nationale pour les opérations étatiques peut considérablement réduire l'acceptation des devises. Selon ces résultats, les politiques publiques pourraient agir comme un instrument de coordination en cas de circulation de plusieurs monnaies.

Sujets : Recherches menées par les banques centrales ; Monnaies numériques

Codes JEL : C92, D83, E40 


\section{Non-Technical Summary}

In many economies, more than one currency circulates. Along the border, national currency often circulates side by side with the neighboring country's currency. More recently, privately issued cryptocurrencies, such as bitcoin, have started being used alongside government-issued money. With multiple objects that can potentially serve the role of payment method, how do people decide which payment method to use?

In this paper, we investigate competition between multiple currencies in a controlled laboratory environment and examine how various features of the economy affect their roles in exchange. The experimental approach complements theoretical and empirical works on multiple currencies. Theories often predict multiple equilibria characterized by different patterns of currency circulation, which weakens their predictive power. Empirical work is sparse because of the lack of micro-data on circulation of multiple currencies. An experimental study allows us to directly observe individuals' currency acceptance decisions, and discern which outcome is likely to emerge.

Our experimental design builds on the two-country, two-currency search model of Matsuyama, Kiyotaki, and Matsui (1993) with indivisible money and indivisible good. The indivisibility framework allows us to abstract away from terms of trade and inflation, and focus on the acceptability of the two currencies. We design four treatments of experiment to study the circulation pattern of the two currencies and investigate how they are affected by economic integration, relative country size, and government policies favoring domestic currency.

We find that subjects' acceptance decisions are little affected by the extent of economic integration. As one country becomes larger, the acceptance rate of foreign currency among its citizens decreases relative to the other country and their counterparts in the treatment with symmetric country sizes. However, in the absence of government transaction policies, the rejection rates for both home and foreign currency are low and tend to decrease over time, providing evidence in favor of selection of the equilibrium where both currencies circulate internationally. The introduction of government transaction policies significantly raises the foreign-currency rejection rate and home bias, pushing the experimental economies toward the national currency equilibrium. 


\section{Introduction}

Government-issued money, or national currency, is the most widely used currency in most modern economies and has an important role as a generally accepted medium of exchange within a nation. Historically, the first known paper currencies were issued by local governments and tended not to circulate beyond a region's borders. Only thereafter did the secondary use of paper currencies from other localities become more common and oftentimes at a lesser scale. While only one or a few international currencies tend to widely circulate at a given point in time, their general acceptance raises the question of whether another currency can substitute or crowd out the use a national currency. More recently, the dominance of national currencies has received renewed interest among policymakers as privately issued cryptocurrencies, such as bitcoin, have started being used alongside government-issued money. What determines which currency out of many potential candidates emerges as a universally accepted payment? And how are circulation patterns affected by government policy?

In this paper, we investigate competition between multiple currencies using experimental methods and examine how various features of the economy affect their roles in exchange. The starting point of our analysis is the two-country, two-currency search model of Matsuyama, Kiyotaki, and Matsui (1993), where domestic and foreign currency compete and can circulate as media of exchange. Search theoretic models are particularly well-suited for studying the use of multiple currencies since these models endogenously generate different payment regimes, without having to restrict which currencies private citizens can accept. For that reason, we adopt a version of Matsuyama, Kiyotaki, and Matsui (1993) in the laboratory to study the circulation pattern of the two currencies and investigate how they are affected by economic integration, relative country size, and government policies favoring domestic currency.

In the two-country, two-currency model, a country is defined by the fact that agents are more likely to encounter compatriots than foreigners. Trade between agents entails an exchange of goods for either home or foreign currency. A currency is modeled as a token object that yields no value if consumed and that cannot be used for production. Since the circulation of these token objects is driven by both fundamentals and beliefs regarding what other agents do, there are multiple equilibria that differ in the areas of circulation of the two currencies. One equilibrium features only local circulation of currencies. In another equilibrium, one currency is internationally accepted while the other remains local. Finally, there is also an equilibrium where both currencies are everywhere accepted. Whether there are zero, one, or two international currencies depends on the fundamentals of the economy, such as country size and degree of economic integration, as well as expectations regarding what other agents do. While the multiplicity of equilibria poses predictive challenges

for the theory, our experimental approach can help discern which equilibrium is selected in the 
laboratory. ${ }^{1}$ Equilibrium selection is therefore an important reason we go to the laboratory.

There are several other advantages of using an experimental approach to study multiple currencies. First, there is lack of micro-level data on the circulation of multiple currencies, which makes empirical studies using field data sparse. ${ }^{2}$ Second, experimental methods give clean control of the environment and allow us to isolate the factors that drive acceptance decisions. Third, we directly observe currency acceptability and can incentivize subjects in the laboratory. Field data often rely on surveys susceptible to errors due to insufficient incentives for truthful or careful reporting, misunderstandings about the survey questions, etc. Finally, it is possible to conduct policy experiments and counterfactuals in the laboratory that are not feasible to implement in practice.

In our benchmark design, we implement a simple version of the model in the laboratory and investigate whether agents who have access to two intrinsically worthless tokens coordinate on an equilibrium with zero, one, or two currencies everywhere accepted. Our design allows us to explore how the degree of economic integration and relative group size matter for currency circulation and equilibrium selection. In the model, these two parameters affect the matching process and hence the likelihood that individuals expect to meet foreigners relative to compatriots.

We introduce three treatments as part of the benchmark design. The baseline treatment features symmetric country sizes and a high level of integration, the second has a lower level of integration and keeps country sizes symmetric, and the third has different country sizes. Results indicate the degree of integration has no significant effect on currency circulation. With asymmetric country sizes, subjects from the larger country show stronger home bias and reject foreign currency more frequently compared with subjects in the baseline treatment. However, the resulting increase in foreign-token rejection rates is not quantitatively large, on average $7.3 \%$ if counting subjects from both groups and $13.8 \%$ if counting only subjects in the larger country.

For the benchmark design, subjects readily accept both currencies even though the matching process is biased towards their home country and other regimes are theoretical possibilities. In addition, the asset distribution across agents in the last period closely matches the theoretical distribution assuming both currencies are universally accepted. This finding is robust across treatments, which suggests integration and relative group size alone are not enough to induce subjects to systematically favor one currency over the other. Convergence to a unified currency regime is also robust to whether there is a fixed or indefinite horizon.

We next extend our design to incorporate government transaction policies biased towards domestic currency. This captures the role of government policies and legal tender laws that aim to increase the acceptance of local money. The basic idea is that by simply accepting a particular

\footnotetext{
${ }^{1}$ There are other papers that adopt an evolutionary approach to study how agents coordinate on an equilibrium; in the context of monetary search models, see e.g., Matsuyama, Kiyotaki, and Matsui (1993) and Wright (1995).

${ }^{2}$ An exception is Colacelli and Blackburn (2009), which provides a micro-level analysis of the circulation of secondary currencies using survey data from Argentina.
} 
currency in its own trades, governments may induce private agents to do the same. ${ }^{3}$ We incorporate these policies in the lab by introducing a subset of agents whose acceptance strategies are hard coded through computerized "robots." Subjects in the lab may therefore be matched with another human subject who makes their own trading decisions, or a robot that follows a publicly announced policy of only accepting its home token. The objective is to determine whether selection still favors universal acceptance when all other currency regimes are candidate equilibria.

We find the presence of government agents coordinates subjects towards rejecting foreign tokens more frequently. The foreign-token rejection rate increases on average from $6.7 \%$ in the baseline treatment to $57 \%$ with government transaction policies. On the other hand, home-token rejection rates remain similar to the baseline treatments without government. These results suggest government policy in the experiment acts as a coordination device towards rejecting foreign currency, consistent with the role of government transaction policies in practice.

The rest of the paper proceeds as follows. Section 2 discusses related literature and our contribution. Section 3 describes the theoretical framework used for the experiments. Section 4 describes the experimental design and outlines the main hypotheses for the experiments. Section 5 reports the main results, discusses robustness, and provides a broader discussion on some modeling and design choices. Section 6 concludes.

\section{Related Literature}

This paper contributes to a growing experimental literature on monetary economics. Here we mention only a few of the most relevant studies; for a more comprehensive survey, see Duffy $(1998,2016)$. The earliest studies on the role of money as medium of exchange in search models are Brown (1996) and Duffy and Ochs (1999). These studies construct experimental tests of the commodity money model of Kiyotaki and Wright (1989). Duffy and Ochs (2002) consider the fiat money model of Kiyotaki and Wright (1993) and find that an intrinsically worthless object can emerge as a medium of exchange. Camera, Noussair and Tucker (2003) conduct an experiment in an overlapping generations economy where fiat money is dominated in rate of return by a dividend-bearing asset, and they identify conditions in which the rate-of-return dominance anomaly can be observed. ${ }^{4}$ Camera and Casari (2014) and Duffy and Puzzello (2014) study monetary exchange versus gift giving when good outcomes can be supported without money through social norms. Their findings suggest that money acts as a coordination device even when it is not theoretically essential for trade. Cam-

\footnotetext{
${ }^{3}$ Aiyagari and Wallace (1997) and Li and Wright (1998) incorporate government transaction policies in the Kiyotaki and Wright (1993) and Trejos and Wright (1995) models, respectively, by introducing government agents who follow exogenous transaction policies regarding which objects they accept as payment. According to the theory, a sufficiently large government sector that only accepts domestic currency can drive foreign currency out of circulation.

${ }^{4}$ In particular, Camera, Noussair and Tucker (2003) find that fiat money is consistently used as a medium of exchange if (1) subjects experienced trading with fiat money prior to the introduction of the dividend-bearing asset, or (2) when the dividend payment occurs after the execuation of trades.
} 
era, Casari, and Bortoletti (2016) and Arifovic, Duffy, and Jiang (2017) study competing payment methods linked to the same currency, with a focus on the effect of the cost and reward structure of different payment methods.

This paper has two key differences from this previous work. First, our experiment studies the circulation patterns of two intrinsically worthless tokens, rather than competition between multiple commodity monies, commodity versus fiat money, gift giving versus fiat money, etc. ${ }^{5}$ Second, we consider an asymmetric matching process where two groups are distinguished by the matching function. This distinction defines an individual's nationality or group membership, a key aspect affecting currency regimes in practice.

The closest papers to ours are Rietz (2017) and Ding and Puzzello (2017), which were both written concurrently with ours. Rietz (2017) studies the acceptance of a secondary currency when a primary currency already circulates based on the dual currency economy of Craig and Waller (2000). Rietz's focus is on the choice to accept the secondary currency given the acceptability of another currency, while we focus on competition between two currencies, distinguish between groups as a result of matching, and introduce a different type of government policy biased towards home currency. Ding and Puzzello (2017) also study competition between two currencies but use the divisible money model of Zhang (2014). Our simpler set-up with indivisible money and goods provides a benchmark that focuses exclusively on the acceptability decision, without having to deal with terms of trade and prices, which may also affect acceptance decisions. While both our studies emphasize the effects of government transaction policies on acceptance decisions, we also study the effects of economic integration and relative country sizes. Our findings are therefore complementary to each other and together offer a more comprehensive picture of the factors (e.g., integration, size, government policies, circulation of a primary currency, information costs, etc.) driving circulation patterns in dual currency economies.

\section{Theoretical Framework}

We base our experiment on a simplified version of the two-country, two-currency search model of Matsuyama, Kiyotaki, and Matsui (1993). We first describe the baseline model without government transaction policies in Section 3.1, and then introduce these policies in Section 3.2.

\footnotetext{
${ }^{5}$ An earlier paper by Arifovic (1997) studies two fiat monies in the Kareken-Wallace overlapping generations economy, with a focus on the behavior of the exchange rate. She finds that both the genetic algorithm simulations and the experiments with human subjects exhibit continuing fluctuations of the exchange rate. Noussair, Plott, and Riezman (2007) study trade and exchange rates using a three-country general equilibrium model where each country issues its own currency and agents are subject to cash-in-advance constraints and must trade with their home currency.
} 


\subsection{Baseline Model without Government Policy}

Time is discrete and continues forever. Agents are divided into two groups, or "countries," called Red and Blue. The measure of agents in country $i \in\{r, b\}$ is $n_{i}$, where $2 n=n_{r}+n_{b}$ is the total size of the economy. All agents have a discount factor across periods of $\beta=1 /(1+r) \in(0,1)$, where $r>0$ is the discount rate.

Countries are defined by a pairwise matching technology where a pair of agents from the same country are more likely to be matched than a pair of agents from different countries. Table 1 summarizes agents' matching probabilities, where entries give $\alpha_{i j}$, the probability an agent from country $i$ meets an agent from $j$. The matching process depends on country sizes, $n_{r}$ and $n_{b}$, and the parameter, $\rho \in[0,1]$, which captures the degree of integration between countries. Other things being equal, it is more likely to meet someone from the larger country. As $\rho \rightarrow 1$, the two countries become more integrated, while $\rho=0$ implies the two economies are closed and do not interact with one another.

Table 1: Meeting Probabilities

\begin{tabular}{lll}
\hline & Red Agent & Blue Agent \\
\hline Red Agent & $\alpha_{r r}=\frac{n_{r}}{n_{r}+n_{b}}+\frac{(1-\rho) n_{b}}{n_{r}+n_{b}}$ & $\alpha_{r b}=\frac{\rho n_{b}}{n_{r}+n_{b}}$ \\
Blue Agent & $\alpha_{b r}=\frac{\rho n_{r}}{n_{r}+n_{b}}$ & $\alpha_{b b}=\frac{n_{b}}{n_{r}+n_{b}}+\frac{(1-\rho) n_{r}}{n_{r}+n_{b}}$ \\
\hline
\end{tabular}

There are three indivisible objects in the economy: a consumption good and two intrinsically worthless tokens. Each agent costlessly produces a different variety of the consumption good but does not want to consume it. Instead, agents get flow utility $u>0$ only from consuming another agent's variety. ${ }^{6}$ Immediately following consumption, agents produce a unit of good at no cost and carry it to the next period. A lack of double coincidence of wants rules out barter. In addition, absence of record keeping precludes cooperative credit arrangements that can be sustained by punishment or reputation. ${ }^{7}$ These assumptions imply that a tangible medium of exchange is needed for trade.

There are two token objects that can potentially serve this role, a red token and a blue token. Both tokens are intrinsically worthless, cannot be used for production, and are only labeled by color. In addition, agents can hold at most one object: one unit of good or one unit of a red or blue token. A fraction $M_{i} \in(0,1)$ of agents (buyers) in country $i$ are initially endowed with an indivisible unit

\footnotetext{
${ }^{6}$ For instance, each agent is specialized in producing a particular variety of a good and does not like to consume his or her own own production variety. Alternatively, as in Diamond (1982), all agents can produce and get flow utility $u>0$ from consuming, but there is a social taboo against consuming one's own production good.

${ }^{7}$ In principle, there could be nonmonetary gift exchange equilibria sustained through community enforcement as in Kandori (1992) and Aliprantis, Camera, and Puzzello (2006). While these gift exchange equilibria are theoretically possible, we do not allow for them in the experiment so that we can focus on the choice between two currencies. For a detailed analysis of gift-giving and monetary equilibria in a different model, see Duffy and Puzzello (2014).
} 
of their home currency, of which there is a constant per capita supply. ${ }^{8}$ The remainder $\left(1-M_{i}\right)$ of agents (sellers) are initially endowed with one unit of good. We assume currency trading is not allowed.

Given the model structure, there are only gains from trade between an agent with currency (buyer) and an agent with good (seller), which considerably shrinks agents' action space and simplifies their decisions. Hence when a buyer and a seller meet, they indicate simultaneously whether or not they want to trade. If both agree, they exchange inventories one for one; otherwise, no trade occurs. Following trade, agents' roles as buyers and sellers are reversed: a buyer immediately consumes the good, earning utility $u>0$, produces one unit of good at no cost and becomes a seller in the next period; similarly, the new owner of currency becomes a buyer.

\subsubsection{Monetary Equilibrium}

The state of the economy is fully characterized by the distribution of tokens. At each point in time, there will be some agents with one unit of money each (buyers) and a disjoint group with no money (sellers). Let $m_{i k}$ denote the fraction of buyers from country $i$ with currency $k$ and $m_{i 0}$ denote the fraction of sellers in country $i$. Since currency is indivisible, the condition

$$
m_{i 0}+m_{i r}+m_{i b}=1
$$

must be satisfied, where the vector $\mathbf{m}=\left(m_{i j}\right)$ describes the asset distribution across agents. In addition, the aggregate supply of currency $i$ in country $i$ must equal its aggregate demand:

$$
n_{i} M_{i}=n_{i} m_{i i}+n_{j} m_{j i} .
$$

We focus on symmetric pure-strategy stationary equilibria, where agents from the same country follow the same pure strategy and asset distributions are constant over time. A buyer who matches with a seller will always want to trade. In what follows, we consider different types of equilibria based on which currencies sellers accept. To simplify presentation, we focus on candidate equilibria where agents always accept their home currency. ${ }^{9}$ The key issue is then whether trade occurs when a seller from $i$ meets a buyer with foreign currency $j \neq i$. Let $\lambda_{i}=1$ if the seller in country $i$ accepts currency $j$ (both domestic and foreign currency circulates in country $i$ ) and $\lambda_{i}=0$ if the seller rejects currency $j$ (only domestic currency circulates in country $i$ ). The regimes we focus on

\footnotetext{
${ }^{8}$ Since a fraction of a country's residents begin with one unit of their home currency, this rules out equilibria where a country's residents only hold the currency issued by the other country. As there is no population growth in the model, the monetary authority issues currency to some fraction of its citizens in the initial period and then shuts down. For our purposes, one can think of the monetary authority in the model as the experimenter.

${ }^{9} \mathrm{We}$ do not impose this restriction in the experiment and only focus on these equilibria here to simplify the presentation; i.e., we allow agents to reject trade when offered a home token. Nonetheless, our experimental results indicate agents almost always accept their home token (between 93 and $97 \%$ of the time when given the opportunity to do so) but not necessarily the foreign token.
} 
are given by $\lambda \equiv\left(\lambda_{r}, \lambda_{b}\right)=\{(0,0),(0,1),(1,0),(1,1)\}$.

We call a currency a national currency if it is only accepted by sellers from that country and a international currency if it is accepted by all sellers. Hence, an equilibrium with two national currencies (no international currency) corresponds to $\lambda=(0,0)$, an equilibrium with one international currency corresponds to $\lambda=\{(0,1),(1,0)\}$, and an equilibrium with two international currencies corresponds to $\lambda=(1,1)$.

When agents of different nationality meet, the stationary distribution of currency holdings satisfy

$$
\begin{aligned}
\alpha_{i j}\left(m_{i 0} m_{j i}-m_{i i} m_{j 0} \lambda_{j}\right) & =0, \\
\alpha_{i j}\left(m_{i 0} m_{j j} \lambda_{i}-m_{i j} m_{j 0}\right) & =0 .
\end{aligned}
$$

These expressions use the fact that trade between agents of the same nationality do not change $\mathbf{m}$ since the aggregate distribution will not be affected. According to (1), when a seller from $i$ meets a buyer from $j$ with currency $i$, the amount of currency $i$ in country $i, m_{i i}$, will increase. At the same time, when a buyer from $i$ with currency $i$ meets a seller from $j, m_{i i}$ will decrease if the agents decide to trade $\left(\lambda_{j}=1\right)$. In a stationary equilibrium, this net change must be zero. A similar explanation applies to (2).

Consequently, the stationary asset distribution $\mathbf{m}$ satisfies

$$
\begin{gathered}
\underbrace{m_{i 0} m_{j i}}_{\text {outflow of currency } i \text { from country } j}=\underbrace{\underbrace{m_{j 0} \lambda_{j}}_{i i}}_{\text {inflow of currency } i \text { to country } j}, \\
\underbrace{m_{i 0} m_{j j} \lambda_{i}}_{\text {inflow of currency } j \text { to country } i}=\underbrace{m_{i j} m_{j 0}}_{\text {outflow of currency } j \text { from country } i}
\end{gathered}
$$

According to (3), the flow out of currency $i$ from country $j$ must equal the flow in of currency $i$ to country $j$. Similarly, (4) says the inflow of currency $j$ to country $i$ must equal the outflow of currency $j$ from $i$.

Let $V_{i 0}$ denote the lifetime utility of a seller from country $i$ who holds no money and $V_{i k}$ denote the lifetime utility of a buyer from country $i$ who starts a period with currency $k$. The flow value of being a seller from country $i$ can be written as

$$
\begin{aligned}
& r V_{i 0}=\underbrace{\left(\alpha_{i i} m_{i i}+\alpha_{i j} m_{j i}\right)\left(V_{i i}-V_{i 0}\right)}_{\text {exp. trade surplus when seller meets buyer with local money }} \\
& +\underbrace{\left(\alpha_{i i} m_{i j}+\alpha_{i j} m_{j j}\right) \lambda_{i}\left(V_{i j}-V_{i 0}\right)}_{\text {exp. trade surplus when seller meets buyer with foreign money }}
\end{aligned}
$$

Equation (5) consists of the probability a seller from $i$ meets a local or foreign buyer holding currency 
$i$ times the trade surplus in that meeting, plus the probability the seller from $i$ meets a local or foreign buyer holding currency $j$.

The flow value of a buyer from $i$ holding domestic currency is

$$
r V_{i i}=\underbrace{\left(\alpha_{i i} m_{i 0}+\alpha_{i j} m_{j 0} \lambda_{j}\right)\left(u+V_{i 0}-V_{i i}\right)}_{\text {exp. trade surplus of buyer with local money in local and foreign meetings }} .
$$

Similarly, the flow value of being a buyer from $i$ holding foreign currency is

$$
r V_{i j}=\underbrace{\left(\alpha_{i i} m_{i 0} \lambda_{i}+\alpha_{i j} m_{j 0}\right)\left(u+V_{i 0}-V_{i j}\right)}_{\text {exp. trade surplus of buyer with foreign money in local and foreign meetings }} .
$$

Equation (6) is the flow value of being a buyer from country $i$ holding domestic currency, which consists of the probability the buyer meets a domestic or foreign seller times the gains from trading. Similarly, equation (7) is the flow value of being a buyer from country $i$ holding foreign currency, which is the probability a buyer meets a domestic or foreign seller times the gains from trading. In (6) and (7), the value of obtaining the consumption good is $u+V_{i 0}$ : one gets direct utility from consumption $(u)$ plus the value of being a seller $\left(V_{i 0}\right)$ since acquiring a consumption good enables the agent to produce next period.

Whether or not trade takes place only depends on the seller's decision to trade since the buyer always wants to trade in any meeting with a seller. Since our focus is on equilibria where sellers always accept their domestic currency, we just need to verify the seller is willing to accept foreign money. In that case, the incentive compatibility conditions for a seller from country $i$ are

$$
\lambda_{i}=\left\{\begin{array}{c}
1 \\
\in[0,1] \text { if } V_{i j}\left\{\begin{array}{l}
> \\
= \\
0
\end{array} V_{i 0} .\right.
\end{array}\right.
$$

According to (8), a seller is willing to accept foreign currency if the value of holding it and becoming a buyer exceeds the value of remaining a seller. For him to reject foreign money, the opposite condition must hold.

\subsubsection{Currency Regimes}

A currency regime is a stationary and symmetric equilibrium, $(\lambda, \mathbf{m}, \mathbf{V})$, satisfying conditions (3)(8). Table 2 summarizes the currency regimes that may emerge (as mentioned earlier, we focus on equilibria where agents always accept home currencies). For each regime, we solve equations (3)-(7) and check the conditions in (8) are satisfied. If so, then the candidate regime constitutes an equilibrium. 
Table 2: Equilibrium Currency Regimes

\begin{tabular}{|c|c|}
\hline Regime & Circulation Pattern \\
\hline \hline$N$ & Two National Currencies \\
$B$ & Blue Currency is International, red Currency is National \\
$R$ & Red Currency is International, blue Currency is National \\
$U$ & Two International Currencies \\
\hline
\end{tabular}

Regime N: Two National Currencies. In this equilibrium, sellers never accept foreign currency: i.e., $\lambda=(0,0)$. We call this Regime $N$. Since only local currency circulates, $m_{r r}=M_{r}$, $m_{b b}=M_{b}$, and $m_{r b}=m_{b r}=0$. The value functions simplify to

$$
\begin{gathered}
r V_{i 0}=\alpha_{i i} M_{i}\left(V_{i i}-V_{i 0}\right), \\
r V_{i i}=\alpha_{i i}\left(1-M_{i}\right)\left(u+V_{i 0}-V_{i i}\right), \\
r V_{i j}=\alpha_{i j}\left(1-M_{j}\right)\left(u+V_{i 0}-V_{i j}\right) .
\end{gathered}
$$

We now verify a seller's incentive condition to never accept foreign currency, or $V_{i j} \leq V_{i 0}$. This will be the case if

$$
\alpha_{i j} \leq \frac{\alpha_{i i}^{2} M_{i}\left(1-M_{i}\right)}{\left(r+\alpha_{i i}\right)\left(1-M_{j}\right)} .
$$

An equilibrium with two national currencies exists so long as $\alpha_{i j}$ is small enough: if one does not come across foreigners too often, then it is optimal to reject foreign money. Equation (9) also gives the existence condition for Regime $N$ in terms of the degree of integration $\rho$ and the size of Red, $n_{r}$. For a given country size, a sufficiently open economy would eliminate this equilibrium since a higher $\rho$ increases the probability of encountering foreigners and thus the incentive to accept foreign money.

Regime R and B: One International Currency. The emergence of an international currency occurs when sellers from one country accept both currencies. Here we focus on the case where sellers from Red accept both currencies, while sellers from Blue only accept domestic money, i.e., $\lambda=(1,0)$. We call this Regime $B$. In that case, the blue token emerges as the sole international medium of exchange. Since the red token only circulates at home: $m_{r r}=M_{r}$ and $m_{b r}=0$. The fraction of blue tokens held in Red and Blue, respectively, are given by

$$
\begin{aligned}
& m_{r b}=\frac{\left(1-M_{r}\right) M_{b}}{1+\frac{\alpha_{b r}}{\alpha_{r b}}\left(1-M_{r}\right)}, \\
& m_{b b}=\frac{M_{b}}{1+\frac{\alpha_{b r}}{\alpha_{r b}}\left(1-M_{r}\right)} .
\end{aligned}
$$


For citizens from Red, the value functions are

$$
\begin{gathered}
r V_{r 0}=\underbrace{\alpha_{r r} m_{r r}\left(V_{r r}-V_{r 0}\right)}_{\text {exp. trade surplus when seller meets buyer from Red with red token }} \\
+\underbrace{\left(\alpha_{r r} m_{r b}+\alpha_{r b} m_{b b}\right)\left(V_{r b}-V_{r 0}\right)}_{\text {exp. trade surplus when seller meets buyer with blue token }} \\
r V_{r r}=\underbrace{\alpha_{r r} m_{r 0}\left(u+V_{r 0}-V_{r r}\right)}_{\text {exp. trade surplus of buyer with local money in local meetings }} \\
\underbrace{\left(\alpha_{r r} m_{r 0}+\alpha_{r b} m_{b 0}\right)\left(u+V_{r 0}-V_{r b}\right)}_{\text {exp. trade surplus of buyer with blue token in local and foreign meetings }}
\end{gathered} .
$$

For citizens from Blue, the value functions are

$$
\begin{gathered}
r V_{b 0}=\left(\alpha_{b r} m_{r b}+\alpha_{b b} m_{b b}\right)\left(V_{b b}-V_{b 0}\right), \\
r V_{b r}=\alpha_{b r} m_{r 0}\left(u+V_{b 0}-V_{b r}\right), \\
r V_{b b}=\left(\alpha_{b r} m_{r 0}+\alpha_{b b} m_{b 0}\right)\left(u+V_{b 0}-V_{b b}\right) .
\end{gathered}
$$

In this equilibrium, $V_{i b}>V_{i 0}$, which guarantees that sellers from both countries have an incentive to accept the blue token. Next, to ensure that sellers from Red are willing to accept the red token, $V_{r r} \geq V_{r 0}$ must hold, which requires

$$
\alpha_{r r} m_{r 0}\left(r+\alpha_{r r} m_{r 0}+\alpha_{r b} m_{b 0}\right) \geq \alpha_{r b} m_{b 0}\left(\alpha_{r r} m_{r b}+\alpha_{r b} m_{b b}\right)
$$

Finally, sellers from Blue will not accept the red token if $V_{b r} \leq V_{b 0}$, which requires

$$
\alpha_{b r} m_{r 0}\left(r+\alpha_{b a} m_{r 0}+\alpha_{b b} m_{b 0}\right) \leq \alpha_{b b} m_{b 0}\left(\alpha_{b a} m_{r b}+\alpha_{b b} m_{b b}\right) .
$$

Intuitively, these conditions require that it be relatively easy for a buyer from Red to find a Red seller, but relatively hard for a Blue buyer to find a Red seller.

Regime U: Two International Currencies. An equilibrium with two international currencies, $\lambda=(1,1)$, occurs when sellers from both countries accept both currencies. We call this Regime $U$. The value functions in this regime are

$$
\begin{gathered}
r V_{i 0}=\left(\alpha_{i i} m_{i i}+\alpha_{i j} m_{j i}\right)\left(V_{i i}-V_{i 0}\right)+\left(\alpha_{i i} m_{i j}+\alpha_{i j} m_{j j}\right)\left(V_{i j}-V_{i 0}\right) \\
r V_{i i}=\left(\alpha_{i i} m_{i 0}+\alpha_{i j} m_{j 0}\right)\left(u+V_{i 0}-V_{i i}\right)
\end{gathered}
$$


Figure 1: Topology of Equilibria



For Regime $U$ to exist, we must have $V_{i i}=V_{i j}$, so that buyers are indifferent between holding currency $i$ and currency $j \neq i$. Consequently, the two monies are perfect substitutes. Since $V_{i i}>V_{i 0}$ is always satisfied, this regime always constitutes an equilibrium.

Figure 1 depicts the typology of the four types of equilibria in $\left(n_{r}, \rho\right)$ space, holding fixed all other parameters: $\beta=1,2 n=10$, and $M_{r}=M_{b}=0.5$. Regime $N$ is an equilibrium if the two economies are not too integrated (the probability of meeting a foreigner is small relative to the probability of meeting a compatriot) and country sizes are not too dissimilar. Regime $R(B)$ is an equilibrium so long as Red (Blue) is not too small. Regime $U$ is always an equilibrium.

\subsection{Model with Government Transaction Policies}

An idea dating back to Smith (1963) and Lerner (1947) is that by accepting a particular currency in its own transactions, governments can induce private citizens to do the same. We examine this idea and introduce a group of traders similar to the government agents in Aiyagari and Wallace (1997) and Li and Wright (1998) who follow an exogenously specified trading rule regarding which currency they accept as payment, rather than choosing to accept a currency based on optimizing behavior. Specifically, we assume there is a constant fraction $g_{b} \in[0,1]$ of government agents in the Red group and a constant fraction $g_{b} \in[0,1]$ in the Blue group.

As before, there are two currencies - red token and blue token - that are identical except for their 
initial distributions. Government agents consume and produce like private agents and are subject to the same matching process. However, in contrast with private agents who adopt trading strategies based on maximizing behavior, government agents follow exogenous trading rules called government transaction policies. In what follows, we consider a benchmark policy where government agents only accept domestic currency in exchange for goods (when a seller), and always accept goods for any currency (when a buyer).

As before, we define currency regimes in terms of the acceptance decisions of private sellers. Hence we continue to call Regime $U$ an equilibrium where both currencies are accepted by private sellers, even though government sellers never accept foreign currency by assumption. The full model set-up and analysis of currency regimes in equilibrium are in Appendix A. Here we summarize the key features and predictions.

In general, the introduction of government agents changes the set of equilibria that exist for a given set of parameters. While the existence condition for a national currencies equilibrium (Regime $N$ ) remains unchanged, the introduction of government agents makes it more difficult to achieve equilibria where foreign currency is accepted. This is because government agents impose a cost to private agents since it may take longer to successfully trade foreign currency for the consumption good. Hence the introduction of government agents slows down total trade and in this way lowers the benefit of accepting foreign currency. As a result, the equilibrium set for Regimes $R, B$, and $U$ shrinks relative to the model without government agents.

\section{Experimental Design}

We now describe our implementation of the model environment in the laboratory. We first discuss our choice of parameters for the experiment, summarize the main hypotheses we evaluate with our design, and finally describe the experimental procedure. Additional details of the design are in Appendices B-E.

\subsection{Model Parameterization}

Our experiments follow a between-subjects design where each session consists of a new group of subjects making decisions under a single parameter set. We conduct four treatments. Table 3 summarizes the parameter values used in each treatment. The combination of parameters for each treatment implies the coexistence of Regimes $N, R, B$ and $U$. There are therefore four types of monetary equilibria that subjects can potentially coordinate on. ${ }^{10}$

For all four treatments, the total number of subjects is $2 n=16$ (except for one session of the LowInt treatment, which has 12 subjects), the fraction of token holders in both groups is

\footnotetext{
${ }^{10}$ There also exists equilibria where one currency is valued while the other is not. These type of equilibria were never observed in our experiment.
} 
$M_{r}=M_{b}=0.5$, and the utility of consuming is $u=10$.

We have three treatments with the benchmark design where government transaction policies are absent $\left(g_{r}=g_{b}=0\right)$. In the Baseline and LowInt treatments, we adopt a symmetric version of the model with equal country sizes $\left(n_{r}=n_{b}=8\right)$, and vary across treatments the degree of integration, $\rho$. Values for $\rho$ are chosen by targeting the probabilities of meeting a foreigner, $\alpha_{r b}$ and $\alpha_{b r}$. With symmetric country sizes, the probability of meeting a foreigner in both countries are the same, i.e., $\alpha_{r b}=\alpha_{b r}$. For the Baseline treatment, $\rho=0.5625$ implies the probability of meeting a foreigner is 0.3 , while for the LowInt treatment, $\rho=0.1875$ implies the probability of meeting a foreigner is 0.1. In the AsySize treatment, $n_{r}=12$ and $n_{b}=4$ (so that the Red group is three times as large as the Blue group). For the AsySize treatment, we set $\rho=0.375$ to obtain $\alpha_{r b}=0.1$ and $\alpha_{b r}=0.3$. In the Govt treatment, there are eight human subjects and eight computerized robots serving as the government agents. We therefore consider a symmetric version of the model where $n_{r}=n_{b}=8$ and $g_{r}=g_{b}=0.5$. Other parameters are set to the same values as in the Baseline treatment.

For all treatments, we consider a limiting case of the model where $\beta \rightarrow 1$. This corresponds to a finite horizon economy with no discounting between periods. Each session of the experiment lasts a fixed 150 periods, which all subjects are fully informed of. There are several reasons why we fix the number of periods instead of implementing an indefinite horizon by random termination. ${ }^{11}$ First, having a fixed number of periods allows for more control and a more commensurate comparison of acceptance rates across groups, sessions, and treatments. In addition, with a fixed horizon, we can guarantee that each session lasts for a sufficient number of periods so that tokens have a chance to circulate across groups. In our set-up, there are two candidates for a medium of exchange that can potentially circulate between two groups, and the economy begins with agents endowed with either the consumption good or home token. If the trading horizon is too short, then subjects will have only limited (and some may have no) encounters with the foreign token. With an indefinite horizon, there is no guarantee against short sequences even if the continuation probability is close to one.

Moreover, the main critique against using a fixed horizon design in a monetary experiment is less of a concern for our study. The critique is that the fixed horizon design eliminates monetary equilibria: if production is costly, agents will choose to produce and trade for money only if they have an opportunity to spend it in the future. ${ }^{12}$ A fixed horizon means that such opportunities

\footnotetext{
${ }^{11}$ The standard approach to implementing infinite horizon with discounting in the laboratory follows Roth and Murnighan (1978). After each period, the experimental economy continues with a fixed probability, set equal to the discount factor, $\beta$. Equivalently, the experimental economy is terminated with probability $1-\beta$.

${ }^{12}$ Given the timing of production, a seller (who already has a unit of good) is indifferent between accepting and rejecting money because production occurred in a previous period and the cost is sunk. Since production occurs before the match, what matters is the incentive in the second to last period, not the final period. In that period, buyers who have consumed have no incentive to produce and need at least two periods to realize the benefit: one period to acquire money and one period to spend it and consume. With only one period left, they cannot reap the benefit, so they will refuse to produce in the second-last period. By backward induction, no production will take place in earlier periods and monetary equilibria unravel.
} 
vanish at the end of the game. By backward induction, agents will not produce from the very beginning of the game. Monetary equilibria unravel as a result. In our experiment, production is costless: subjects are endowed with one unit of good after consumption. ${ }^{13}$ When production is costless, agents have no strict incentive to refuse to produce even when the game is about to end, so monetary equilibria can be sustained. ${ }^{14}$

Before settling on the final design, we ran pilot sessions to compare the design with a fixed horizon versus an indefinite horizon. We ran five sessions of the same design except for the trading horizon: two sessions had a definite horizon of 100 periods, and three had an indefinite horizon. ${ }^{15}$ For the latter, subjects were instructed the number of periods was determined by a random termination device and there is a $\beta=99 \%$ chance the experiment would continue to another period. We used predrawn random sequences and the three sessions lasted for 110, 116 and 102 periods, respectively. We find that overall, token rejection rates were higher for the indefinite horizon sessions. Specifically, the foreign (home) rejection rate for the three indefinite horizon sessions (averaged across all individual subjects in these sessions) was $16.1 \%$ (6.6\%), versus $4.2 \%$ (3.7\%) for the two definite horizon sessions. We would expect to see lower rejection rates for the finite horizon sessions if there were end-of-period effects; the fact that we observed the opposite helps alleviate this concern. We also find that the standard deviation of individual rejection rates was higher than for the finite horizon sessions. The standard deviation of the foreign- (home-) token rejection rate was $24.5 \%$ (16.1\%) for the indefinite horizon sessions, compared with $7.1 \%(6.3 \%)$ for the definite horizon sessions. This suggests that uncertainty in the trading horizon is partly inducing higher rejection rates and more heterogeneity across subjects' actions (and therefore noisier aggregate results).

\footnotetext{
${ }^{13}$ We recognize that with costless production, there is also an equilibrium where agents give their production for free without asking for money. We rule out this equilibrium by making assumptions that some currency must be used to trade so that we can focus on the choice between two fiat currencies. We also recognize that although the assumption of costless production means that monetary equilibria can be sustained, it does not remove other possibilities: in the final period, sellers are indifferent between trading and not trading, and in theory, can choose to trade with any probability. By backward induction, agents' choices in the final period can affect their decisions in earlier periods. This concern did not materialize in our experiment. While we analyze the experimental data, we investigate how the choices at the end of the game affect earlier decisions, and we find the effect is in general statistically insignificant and quantitatively small (the results are reported in Tables D3 and D4 in Appendix D).

${ }^{14}$ With costly production and a fixed horizon, one way to sustain monetary equilibria is to automate production, instead of modeling it as a choice. Agents will have no strict incentive to reject money in the final period as long as the production cost is less than the utility from consumption.

${ }^{15}$ The design of the pilot sessions is similar to the Baseline except for the horizon and the probability of meeting a foreigner, set to 0.25 , slightly lower than the value of 0.3 used in the Baseline. We decided to use 0.3 in our final design since it makes the meeting probabilities slightly easier to communicate, especially with the AsySize treatment (if we use 0.25 for the Baseline, then $\alpha_{b r}$ would be 0.083 [8.3\%] for the Asysize treatment). We also increased the duration of the experiment from 100 to 150 periods to get more observations on the decision to accept foreign tokens.
} 


\subsection{Hypotheses}

We use the model predictions to form hypotheses on expected findings in the lab. Hypothesis 1 concerns the home-token rejection rate and Hypotheses 2-4 conjecture the effect of the treatment variables in two parts: part $(a)$ is of weak form on the change in foreign-token rejection rates and degrees of home bias, defined as \% Foreign Token Rejections - \% Home Token Rejections, and part (b) is of strong form regarding equilibrium selection.

Hypothesis 1. The home-token rejection rate is lower than the foreign-token rejection rate and will remain constant across treatments.

Hypothesis 2. As the economy becomes less integrated (Baseline to LowInt),

(a) the foreign-token rejection rate and home bias will increase,

(b) the economy will move from the unified currency equilibrium (Regime $U$ ) to the national currency equilibrium (Regime $N$ ).

Hypothesis 3. As the size of the Red group increases relative to the Blue group (Baseline to AsySize),

(a) the foreign-token rejection rate and home bias by agents in the Red group will increase; the foreign-token rejection rate and home bias by agents in the Blue group will remain constant, (b) the economy will move from the unified currency equilibrium (Regime $U$ ) to an equilibrium where the red currency is the only international currency (Regime $R$ ).

Hypothesis 4. With government agents (Baseline to the Govt treatment),

(a) the foreign-token rejection rate and home bias by agents in both groups will increase,

(b) the economy will move from the unified currency equilibrium (Regime $U$ ) to the national currency regime (Regime $N$ ).

\subsection{Description of the Experimental Procedure}

In this study, all experimental sessions were conducted at the Vernon Smith Experimental Economics Laboratory at Purdue University in 2015 and 2016. Participants were undergraduate students at Purdue University, recruited through the Online Recruitment System for Economics Experiments. No subject participated in more than one session of the project, although some subjects participated previously in other economics experiments run by other researchers. The total length of a session ranged from 50 to 80 minutes. Participants received a $\$ 5$ participation payment plus earnings from the experiment. Total earning from the experiment ranged from $\$ 9$ to $\$ 18$ per subject, with median earnings of $\$ 14$. 
Each session consisted of instructions, a follow-up quiz, and the experiment. Upon entering the laboratory, participants were randomly assigned a computer station and given a written copy of the instructions. The instructions were then read out loud by the experimenter to reinforce the common knowledge nature of the environment. Participants then completed a short quiz about the instructions. After completing the quiz, the experimenter went over the correct answers, answered questions, collected the quiz, and began the experiment. All parts of the experiment were conducted with z-Tree (Fischbacher 2007).

Each session consists of 150 periods of bilateral interactions with random matching. Participants are divided into one of two groups, Red or Blue, of potentially different sizes. Group membership is randomly assigned and remains fixed throughout the session. Each participant is initially endowed with one unit of a consumption good (called "corn" in the experiment) one unit of a blue token, or one unit of a red token. This implies an individual's role as a buyer or seller in the first period is exogenous and determined by random assignment; however, in subsequent periods, an individual's role as buyer or seller is endogenously determined by trading outcomes in the previous period.

While tokens are intrinsically worthless objects and cannot be redeemed for points, holding corn yields positive points if and only if one obtains it from someone else. To receive points, individuals must trade either a blue or red token for corn. The instructions also emphasized that it may take more than one period to obtain corn from another participant. Since tokens and corn are indivisible, exchanges are one for one. Participants are induced to maximize their earnings in points, which are converted into U.S. dollars and paid out at the end of the experiment. For all sessions, we used an exchange rate of 1 point $=\$ 0.02$.

In each period, a random matching process pairs an individual with a partner. All participants are informed of the ex ante meeting probabilities in the instructions. As in the model, the probability an individual is paired with someone from their own group is greater than the probability of being paired with someone from the other group. These probabilities differ across treatments. For instance, in the AsySize treatment, subjects are instructed:

In each period, you may either be matched with someone from your own group, or someone from the other group.

- If you are in Group Red, there is a 90\% chance you will be paired with someone else from Group Red and a 10\% chance you will be paired with someone from Group Blue.

- If you are in Group Blue, there is a $70 \%$ chance you will be paired with someone else from Group Blue and a 30\% chance you will be paired with someone from Group Red.

These probabilities will remain the same throughout the experiment and will appear on your computer screen.

Once matched, both parties find out the good their partner holds. If one subject holds corn and the other holds token, the pair is simultaneously asked in isolation whether they would like 
Figure 2: Timing of the Experiment

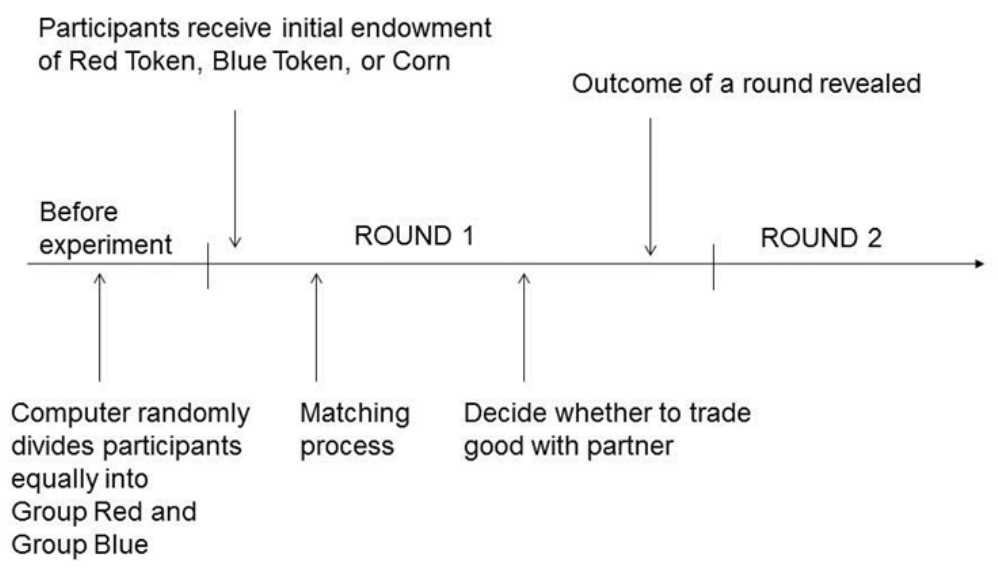

to exchange inventories with their partner. Trade occurs if and only if both parties agree, and only someone who successfully trades for corn will be awarded points. If trade occurs, each subject begins the next period holding their partner's good from the previous period. If both subjects hold corn or both hold tokens, then they are informed about the situation. No trade occurs and they carry their current inventory into the next period. After completing a period, subjects are rematched for the next period and subjects are asked again if they would like to trade. Figure 2 summarizes the timing of the experiment and Figure 3 is a sample screenshot of the trading screen for the Govt treatment.

\section{Experimental Results}

We now report the main findings from the 16 sessions described in the previous section. Table 4 summarizes aggregate rejection rates of foreign and home tokens and the degree of home bias averaged across all subjects. Table 5 reports the same variables by group.

In addition, we run probit regressions with random effects on the rejection rate in foreign tokencorn and home token-corn meetings. Variables in the probit regressions are defined in Table 6 . We run three sets of regressions. The first is for the rejection rate in home token-corn meetings, and the second for the rejection rate in foreign token meetings, and the third for home bias. Each set includes 15 regressions. The first three use data from all four treatments with observations for all players, players in the Red group, and players in the Blue group, respectively. The rest use data from each of the four individual treatments. In the first two sets of regressions, we include the variable "Period" to capture the time trend of the rejection rate. The effects of the integration parameter, asymmetric country sizes, and government transaction policies are captured respectively by the 
Figure 3: Trading Screen (Govt Treatment)



coefficients on the dummy variables, "LowInt," "AsySize," and "Govt." The same variables appear in the third set of regressions; in addition, we include product terms of these variables multiplied by "Foreign." The time trend of home bias is captured by the coefficient on "Period x Foreign." The effect of the treatment variables on home bias is captured by the product terms "LowInt $\mathrm{x}$ Foreign," "AsySize x Foreign," and "Govt x Foreign." The regression results are summarized in Tables 7-9. For each variable, we report its marginal effects on the percentage of token rejections, the standard error, the t-statistic, and the $p$-value. ${ }^{16}$

Finding 1 (Home-Token Rejection Rate). The home-token rejection rate is low and decreases over time in all four treatments and for both groups. In addition, the rejection rate is not significantly affected by any of the treatment variables.

Finding 1 is consistent with Hypothesis 1. The home rejection rate averages $3.1 \%$ for the Baseline treatment, $5.5 \%$ for the LowInt treatment, $7.0 \%$ for the AsySize treatment, and $4.4 \%$ among human subjects in the Govt treatment. ${ }^{17}$ Table 4 also shows that the home rejection rate decreases with time. The home-token rejection rate decreases for all four treatments from the first half to the

\footnotetext{
${ }^{16}$ For robustness, we rerun the regressions on home and token rejection rates on time dummies in place of the linear time trend term "Period." We divide the 150 periods into 15 segments with 10 periods each and create a dummy for each segment, and use 14 of them in the robustness-check regressions. In Tables D1 and D2 in Appendix D, we report the coefficients, standard errors, and t-statistics on the three treatment variables for regressions using data from all four treatments. The results are very close to those from the regressions with the linear time trend.

${ }^{17}$ The only session with a relatively high home-token rejection rate (17.8\%) is the last session of the AsySize
} 
second half of the experiment: from $5.3 \%$ to $1.0 \%$ (Baseline), $8.1 \%$ to $2.4 \%$ (LowInt), $8.7 \%$ to $5.2 \%$ (AsySize), and $6.9 \%$ to $2.0 \%$ (Govt). ${ }^{18}$ The regression results in Table 7 confirm the decreasing trend in home-token rejection rates. In all 15 regressions, the coefficients on "Period" are negative and statistically significant at the $10 \%$ significance level (and at the $1 \%$ level in 12 regressions). Pooling data from all sessions, we find that the home-token rejection rate decreases by $9.8 \%$ from the first to 150th period.

Finally, the home-token rejection rate is not significantly affected by the treatment variables. From regressions (1)-(3) in Table 7, the only significant coefficient at the $10 \%$ level is the one on "LowInt" for the Blue group. Even there, the magnitude is small at $3.1 \%$.

Finding 2 (Effect of Integration on Foreign-Token Rejection Rate and Home Bias). A lower degree of integration has a weak positive effect on the foreign-token rejection rate and the degree of home bias, both of which attenuate over time.

The LowInt treatment features a lower probability of meeting foreigners at $10 \%$ compared with $30 \%$ in the Baseline treatment. In Hypothesis 2, we conjecture that foreign-token rejection rates and home bias will increase as the two economies become less integrated. The experimental results provide some, albeit weak, evidence for this hypothesis. From Table 4, the rejection rate for foreign tokens and the degree of home bias are higher for the LowInt treatment. Across all subjects and all four sessions, the aggregate rejection rates for foreign tokens are $6.7 \%$ (Baseline) and $11 \%$ (LowInt). The aggregate degrees of home bias are $3.6 \%$ (Baseline) and 5.5\% (LowInt). ${ }^{19}$ However, the differences between the Baseline and LowInt treatment are small in magnitude and tend to dissipate over time. In the second half of the experiment, the foreign-token rejection rates are $6.4 \%$ (LowInt) versus $4.0 \%$ (Baseline). Similarly, in the second half of the experiment, home bias is only slightly higher $4.0 \%$ (LowInt) versus $3.0 \%$ (Baseline).

The weak effect of integration on token rejection rates is somewhat surprising. One would expect individuals are more willing to accept foreign currencies when they interact with foreigners more frequently. For example, those residing along the geographical border between two countries may be more willing to accept foreign currency. A possible reason for the discrepancy in our findings is that the extent of economic integration has more aspects in reality such as the recognizability

treatment (Session 12). This is mainly due to one subject, who chose to always reject the home token in all 70 corn-home token meetings. In Session 12, the total number of home token rejections is 79 . The same subject also chose to always reject the foreign token in all 14 corn-foreign token meetings. If we exclude this subject from our calculation, the home rejection rate for session 12 is $2.4 \%$, and the average for AsySize treatment is $3.1 \%$.

${ }^{18}$ Again, the relatively high rejection rate for treatment AsySize is due to one subject in session 12 . If we exclude this subject, then the home rejection rate for the AsySize treatment will decrease from $5.3 \%$ in the first half to $0.8 \%$ in the second half of the experiment.

${ }^{19}$ The higher foreign-token rejection rate and home bias in the LowInt treatment is mainly driven by the Blue group. The coefficients on "LowInt" are $6.7 \%$ in the regression for foreign-token rejection rate and $5.1 \%$ in the regression for home bias for the Blue group, and both are significant at the $10 \%$ significance level. For the Red group, the coefficients are statitically insignificant with large $p$-values. 
of the foreign currency, costs of maintaining a foreign currency account, etc. In our experiment, economic integration is captured purely by the probability of meeting foreigners.

\section{Finding 3 (Effect of Relative Group Size on Foreign-Token Rejection Rates and Home}

Bias). As the size of one group increases, foreign-token rejection rates and home bias increase for subjects in the larger group, while remain roughly constant for subjects in the smaller group.

Compared with the Baseline treatment, the AsySize treatment features asymmetric country sizes and different probabilities of meeting foreigners for the Red and Blue groups. The larger (Red) group has 12 subjects versus 4 subjects in the smaller (Blue) group, and individuals meet foreigners with a smaller probability of $10 \%$ versus $30 \%$ for the Blue group. Hypothesis 3 conjectures the Red group will have a higher foreign-token rejection rate and home bias while the Blue group will not, which is validated by Finding 3 .

The aggregate rejection rate for foreign tokens is $14.0 \%$ in the AsySize treatment versus $6.7 \%$ Baseline treatment. The higher rejection rate is mainly driven by subjects from the larger country: the average foreign-token rejection rate is $19.9 \%$ for the Red group versus $7.7 \%$ for the Blue group (and $6.1 \%$ for the Red group in the Baseline treatment).

The probit regressions confirm these findings. In Table 8, the coefficient on "AsySize" in the pooled regression is $6.9 \%$ and significant at the $5 \%$ level; i.e., the rejection rate for foreign tokens in the AsySize treatment is $6.9 \%$ higher than in the Baseline. This higher rejection rate mainly comes from subjects in the Red group. The coefficient on "AsySize" is $9.1 \%$ and significant at the $5 \%$ level in the regression with observations on the Red group, and not statistically significant for the Blue group. Furthermore, the foreign-token rejection rate for the Red group does not significantly decrease over time: the coefficient on "Period" in regression 11 in Table 8 has a $p$-value of $16 \%$. Meanwhile, the foreign-token rejection rate for the Blue group falls across time: the coefficient on "Period" in regression 12 in Table 8 is negative and significant at the $10 \%$ level.

Home bias follows a similar pattern. From regression 2 in Table 9, relative to the Baseline treatment, the degree of home bias for the Red group in the AsySize treatment increases by $8.14 \%$, captured by the coefficient on "AsySize X Foreign," which is statistically significant at the $1 \%$ significance level. The coefficient is not statistically significant for the Blue group. In addition, the level of home bias does not change significantly over time for the Red group (the coefficient on "Period" in regression 11 in Table 9 has a $p$-value of 14\%), but decreases for the Blue group.

Finding 4 (Effect of Government Transaction Policies on Foreign-Token Rejection Rate and Home Bias). Government transaction polices significantly increase foreign-token rejection rates and home bias.

Our results support our conjecture in Hypothesis 4 that introducing government agents who accept only home tokens will increase foreign-token rejection rates and home bias among private 
agents. From Table 8, foreign-token rejection rates increase considerably with the introduction of government policies, i.e., the aggregate foreign-token rejection rate increases to $57.4 \%$ from $6.7 \%$ in the Baseline treatment. From regression 1 in Table 8, government transaction policies increase the foreign-token rejection rate by $29.8 \%$. In addition, the foreign-token rejection rate does not change much over time.

Similarly, the degree of home bias increases from $3.6 \%$ in the Baseline treatment to $52.9 \%$ with the introduction of government policies. From the probit regressions in Table 9, such policies increase home bias by $17.3 \%$. Further, the relatively high degree of home bias in the Govt treatment tends to persist over time. Taken together, Findings $2-4$ indicate a strong effect of government policies on token rejection rates.

Finding 5 (Equilibrium Selection). The experimental economies without government policies converge close to the unified currency regime. The experimental economies in the Govt treatment lie approximately half way between the unified currency regime and the national currency regime.

Finding 5 examines the strong form of Hypotheses 2-4 on equilibrium selection where we conjecture that the experimental economy will be close to the uniform currency regime in the Baseline treatment, the national currency regime in the LowInt treatment, the regime with the red token as the single international currency in the AsySize treatment, and the national currency regime in the Govt treatment.

To gain more insight on selection, Figure 4 shows the histogram of token rejection rates in the second half of the experiment. The two horizontal axes represent the home-token and foreign-token rejection rates, respectively. Each axis runs from $0 \%$ to $100 \%$ and is split into 20 intervals of equal lengths. This generates 21 bins along each axis, with the last bin being a point at $100 \%$. Then, for each treatment, we sort subjects into these bins according to their rejection rates (there are 64 subjects in the first three treatments and 32 in the Govt treatment).

The results suggest that with a high level of integration, symmetric country sizes, and absence of government transaction policies, the experimental economy indeed selects the uniform currency regime. As described earlier, both the home- and foreign-token rejection rates in the Baseline treatment are low and tend to decrease over time. In the second half of the experiment, the hometoken rejection rate is $1.0 \%$ and the foreign-token rejection rate is $4.0 \%$. From Figure 4 , there is a spike at $73.4 \%$ in the $(<5 \%,<5 \%)$ bin, and $79.7 \%$ of subjects reject foreign and home tokens less than $10 \%$ of the time. In other words, the majority of subjects have very low rejection rates.

Our initial conjecture that a lower level of economic integration will move the economy to the national currency regime is not strongly supported in the experimental data. As discussed earlier, there is little change in terms of token rejection rate in the LowInt treatment relative to the Baseline treatment. In the second half of the experiment, the rejection rate is $4.0 \%$ for home tokens and 
$6.4 \%$ for foreign tokens. Again, there is a spike at $66.7 \%$ in the $(<5 \%,<5 \%)$ bin, and $78.3 \%$ of subjects reject foreign and home tokens less than $10 \%$ of the time. These results suggest the experimental economies stay close to the uniform currency regime in the LowInt treatment.

Increasing the size of the Red group relative to the Blue group does move the economy toward the regime with the red token being the only international currency. However, the experimental economies are still far away from that equilibrium, and instead stay much closer to the uniform currency regime. In the second half of the experiment, the foreign-token rejection rate is $16.9 \%$ for the red group, the group with a higher foreign-token rejection rate. ${ }^{20}$ The histogram for the Red group in the AsySize treatment remains similar to the first two treatments, with a spike at $68.8 \%$ in the $(<5 \%,<5 \%)$ bin, and $77.1 \%$ of subjects reject foreign and home tokens less than $10 \%$ of the time.

Further evidence that the experimental economies in the first three treatments are close to the unified currency regime can be seen by examining the asset distribution among subjects. Figures $5 \mathrm{a}-\mathrm{c}$ graph the asset distribution for each of the four experimental sessions together with one simulated session assuming that both currencies are international for the first three treatments (one treatment per page). The black line represents the percentage of agents holding corn, and the red line the percentage of agents holding the red token, and the blue line the percentage of agents holding the blue token. The percentages are calculated as the average of all past periods. The upper graphs are for the Red group, and the lower graphs are for the Blue group. From Figures $4 \mathrm{a}-\mathrm{c}$, the distribution in the first three treatments closely matches the theoretical simulated asset distributions assuming both currencies are international. Table 10 reports the asset distribution for each session (averaged across the entire duration of the experiment), the treatment average, and the average across 50 simulated sessions assuming both currencies are international. The experimental and simulated distributions are very similar.

In the Govt treatment, the foreign-token rejection rate and degree of home bias are both higher and more persistent than in the first three treatments. In the second half of the experiment, the foreign-token rejection rate is $55.0 \%$ and the degree of home bias is $53.0 \%$, which suggests the experimental economies lie about half way between the unified currency equilibrium (with $0 \%$ home bias) and national currency equilibrium (with $100 \%$ home bias). Unlike the histograms of the first three treatments, there is are more cross-sectional differences among subjects in foreign-token rejection rates. There are two spikes in the histogram: $25.0 \%$ subjects reject both home and foreign tokens less than $5 \%$ of the time, and $28.1 \%$ reject home tokens less than $5 \%$ of the time, but always reject the foreign token.

\footnotetext{
${ }^{20}$ If we exclude the subject who always rejected tokens, the foreign-token rejection rate for the Red group goes down to $12.6 \%$.
} 


\section{Conclusions and Future Research}

In this paper, we investigate currency competition in the laboratory. The framework for our experiment is a simple two-country, two-currency random matching model where a domestic and foreign currency can compete as media of exchange. As a result of strategic externality, a key feature of the theory is the presence of multiple equilibria featuring zero, one, or two international currencies. The experimental method allows us to study which equilibrium is selected by human subjects, and how the degree of economic integration, the relative country size, and government transaction policies favoring domestic currency affect the acceptance decisions by private agents.

We find that subjects' acceptance decisions are little affected by the extent of economic integration. As one country becomes larger, the acceptance rate of foreign currency among its citizens decreases relative to the other country and their counterparts in the treatment with symmetric country sizes. However, in the absence of government transaction policies, the rejection rates for both home and foreign currency are low and tend to decrease over time, providing evidence in favor of selection of the equilibrium where both currencies circulate internationally. The introduction of government transaction policies significantly raises the foreign currency rejection rate and home bias, pushing the experimental economies toward the national currency equilibrium.

Our study is based on the indivisible money, indivisible good framework and abstracts away from currency exchange and inflation. The assumption of indivisible goods can be relaxed, as in Shi (1995) and Trejos and Wright (1995, 1996), which makes it possible to determine prices and exchange rates. Similarly, the assumption of indivisible money can also be relaxed, as in Shi (1997) and Lagos and Wright (2005), which makes it possible to have money growth and inflation. While it is clearly desirable to have a model with these features, we adopt this simpler specification first in order to focus on the acceptability of the two currencies, holding prices fixed and abstracting from inflation. An interesting and promising direction for future work is to explore these topics in the context of a divisible money, divisible good framework, as in Ding and Puzzello (2017), but with a role for money growth and monetary policy. 


\section{References}

Aiyagari, Rao and Neil Wallace (1997). "Government Transaction Policy, the Medium of Exchange, and Welfare." Journal of Economic Theory, 74, 1-18.

Aliprantis, Charalambos, Gabriele Camera, and Daniela Puzzello (2006). "Matching and Anonymity." Economic Theory, 29, 415-432.

Arifovic, Jasmina (1997). "The Behavior of the Exchange Rate in the Genetic Algorithm and Experimental Economies." Journal of Political Economy, 104, 510-541.

Arifovic, Jasmina, John Duffy, and Janet Hua Jiang (2017). "Adoption of a New Payment Method: Theory and Experimental Evidence." Bank of Canada Staff Working Paper 2017-28.

Brown, Paul (1996). "Experimental Evidence on Money as a Medium of Exchange." Journal of Economic Dynamics and Control, 20, 583-600.

Camera, Gabriele and Marco Casari (2014). "The Coordination Value of Monetary Exchange: Experimental Evidence." American Economic Journal: Microeconomics, 6, 629-660.

Camera, Gabriele, Marco Casari, and Stefania Bortolotti (2016). "An Experiment on Retail Payment Systems." Journal of Money, Credit, and Banking, 48, 363-392.

Camera, Gabriele, Charles N. Noussair, and Steven Tucker (2003). "Rate-of-Return Dominance and Efficiency in an Experimental Economy." Economic Theory, 22, 629-660.

Colacelli, Mariana and David J.H. Blackburn (2009). "Secondary Currency: An Empirical Analysis." Journal of Monetary Economics, 56, 295-308.

Craig, Ben and Christopher Waller (2000). "Dual-Currency Economies as Multiple-Payment Systems." Quarterly Review, 45(1), 155-184.

Diamond, Peter (1982). "Aggregate Demand Management in Search Equilibrium." Journal of Political Economy, 90, 881-894.

Ding, Shuzhe and Daniela Puzzelo (2017). "Legal Restrictions and International Currencies: An Experimental Approach." Working Paper.

Duffy, John (1998). "Monetary Theory in the Laboratory." Federal Reserve Bank of St. Louis, Economic Review, 9-26.

Duffy, John (2016). "Macroeconomics: A Survey of Laboratory Research." In Macroeconomics: A Survey of Laboratory Research, edited by Kagel, John H., and Alvin E. Roth, Princeton University Press.

Duffy, John and Jack Ochs (1999). "Emergence of Money as a Medium of Exchange: An Experimental Study." American Economic Review, 89(4): 847-877.

Duffy, John and Jack Ochs (2002). "Intrinsically Worthless Objects as Media of Exchange: Experimental Evidence." International Economic Review, 43, 637-673. 
Duffy, John and Daniela Puzzello (2014). "Gift Exchange versus Monetary Exchange: Experimental Evidence." American Economic Review, 104(6), 1735-1776.

Fischbacher, Urs (2007). "Z-Tree: Toolbox for Ready-made Economic Experiments." Journal of Experimental Economics, 10, 171-178.

Kandori, Michihiro (1992). "Social Norms and Community Enforcement." Review of Economic Studies, 59(1), 63-80.

Kiyotaki, Nobuhiro and Randall Wright (1989). "On Money as a Medium of Exchange." Journal of Political Economy, 97, 927-954.

Kiyotaki, Nobuhiro and Randall Wright (1993). "A Search-Theoretic Approach to Monetary Economics." American Economic Review, 83(3), 63-77.

Lagos, Ricardo and Randall Wright (2005). "A Unified Framework for Monetary Theory and Policy Analysis." Journal of Political Economy, 113(3), 463-486.

Lerner, Abba (1947). "Money as Creature of the State." American Economic Review, 83, 312217.

Li, Yiting and Randall Wright (1998). "Government Transaction Policy, Media of Exchange, and Prices." Journal of Economic Theory, 81(2), 290-313.

Matsuyama, Kiminori, Nobuhiro Kiyotaki, and Akihiko Matsui (1993). "Toward a Theory of International Currency." Review of Economic Studies, 60, 283-307.

Noussair, Charles, Charles Plott, and Raymond Riezman (2007). "Production, Trade, Prices, Exchange Rates and Equilibration in Large Experimental Economies." European Economic Review, 51, 49-76.

Rietz, Justin (2017). "Secondary Currency Acceptance: Experimental Evidence with a Dual Currency Search Model." Mimeo.

Roth, Alvin and Keith Murnighan (1978). "Equilibrium Behavior and Repeated Play of the Prisoner's Dilemma." Journal of Mathematical Psychology, 17(2), 189-198.

Shi, Shougong (1995). "Money and Prices: A Model of Search and Bargaining," Journal of Economic Theory, 67(2), 467-496.

Shi, Shouyong (1997). "A Divisible Search Model of Fiat Money," Econometrica, 65(1), 75-102.

Smith, Adam (1963). The Wealth of Nations, Richard D. Irwin Inc., Homewood.

Trejos, Alberto and Randall Wright (1995). "Search, Bargaining, Money, and Prices." Journal of Political Economy, 103(1), 118-141.

Trejos, Alberto and Randall Wright (1996). "Search-Theoretic Models of International Currency." Federal Reserve Bank of St. Louis Review, 78(3), 117-132.

Wright, Randall (1995). "Search, Evolution, and Money." Journal of Economic Dynamics and Control, 19, 181-206.

Zhang, Cathy (2014). "An Information-Based Theory of International Currency." Journal of International Economics, 93(2), 286-301. 
Figure 4: Histogram of Token Rejection Rates (Rounds 76-150)
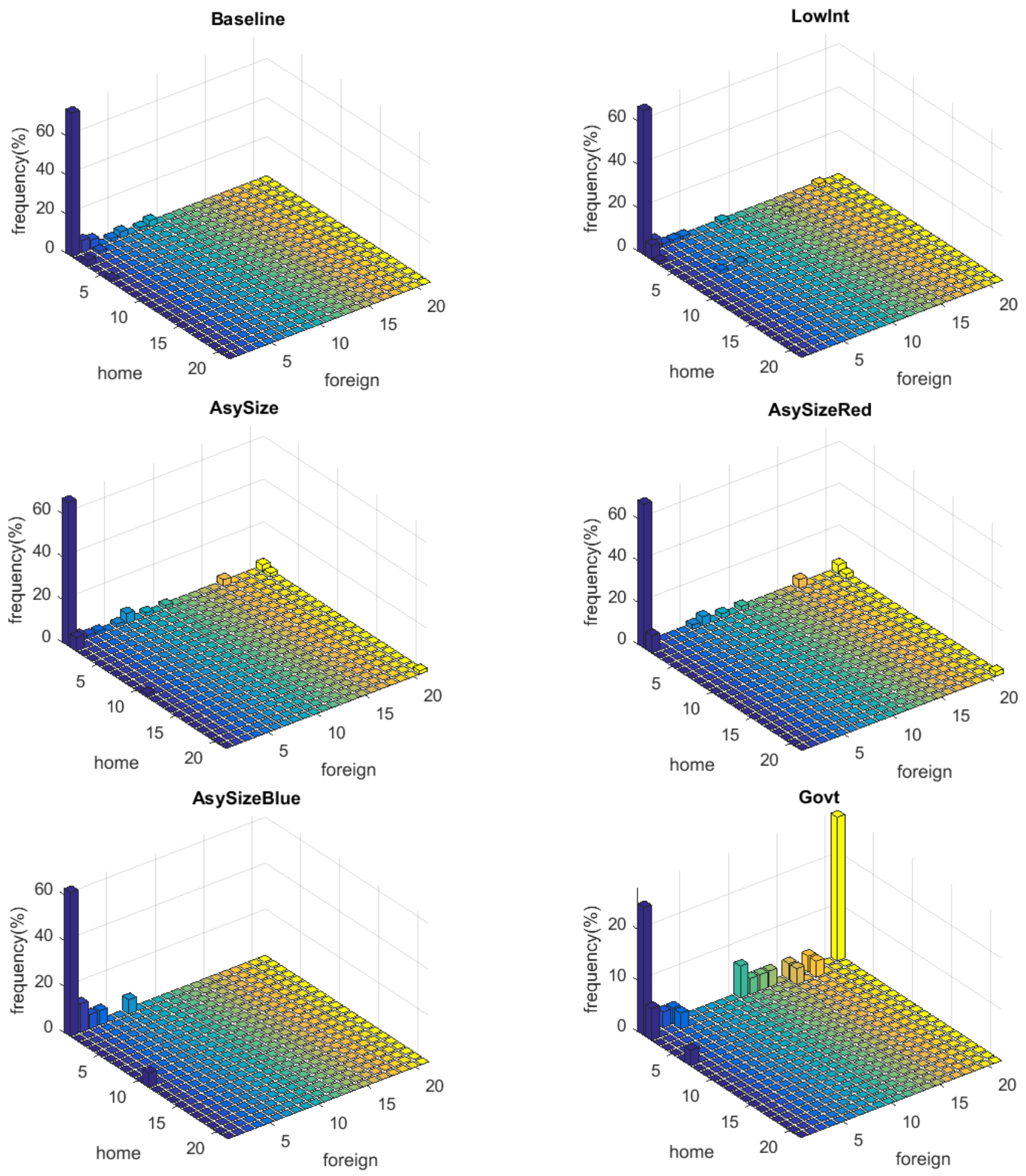

Notes. The figure graphs the two-variable histogram of individual subjects' token rejection rates in the second half of the experiment. The vertical axis is in percentage. There are 21 bins each for home-token and foreigntoken rejection rates. The range of the $1^{\text {st }}$ bin is $[0,5 \%)$, the $2^{\text {nd }}[5 \%, 10 \%)$, and so on. The last $\left(21^{\text {st }}\right)$ bin is a point $100 \%$. For the AsySize treatment, we graph the histogram for all subjects together, the Red group and the Blue group. For the other three treatments, we graph the histogram for all subjects together. 
Figure 5a: Baseline Asset Distribution - Experimental Sessions and Simulation
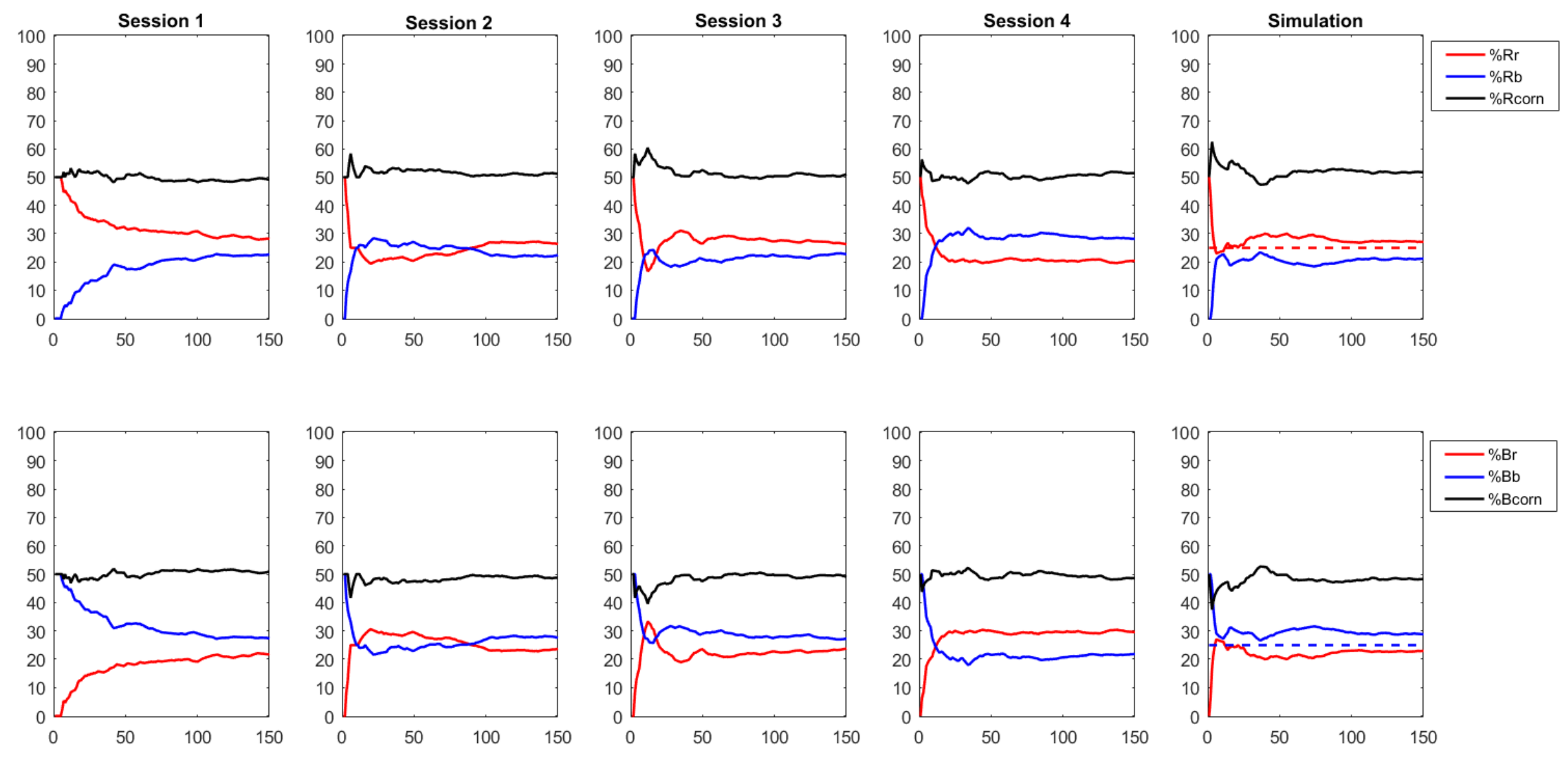

Notes. (1) The black line is the percentage of agents holding corns, the red line the percentage of agents holding red tokens, and the blue line the percentage of agents holding blue tokens. (2) The upper panels are the distribution for the Red group, and the lower panels are for the Blue group. The straight line in the last column of graphs represents the percentage of Red agents holding red tokens, and Blue agents holding blue tokens assuming a continuum of agents. 
Figure 5b: LowInt Asset Distribution - Experimental Sessions and Simulation


Notes. (1) The black line is the percentage of agents holding corns, the red line the percentage of agents holding red tokens, and the blue line the percentage of agents holding blue tokens. (2) The upper panels are the distribution for the Red group, and the lower panels are for the Blue group. The straight line in the last column of graphs represents the percentage of Red agents holding red tokens, and Blue agents holding blue tokens assuming a continuum of agents. 
Figure 5c: AsySize Asset Distribution - Experimental Sessions and Simulation


Notes. (1) The black line is the percentage of agents holding corns, the red line the percentage of agents holding red tokens, and the blue line the percentage of agents holding blue tokens. (2) The upper panels are the distribution for the Red group, and the lower panels are for the Blue group. The straight line in the last column of graphs represents the percentage of Red agents holding red tokens, and Blue agents holding blue tokens assuming a continuum of agents. 
Table 3: Treatments and Parameters

\begin{tabular}{lllllllll}
\hline \hline Treatment & $n r$ & $n b$ & $g$ & $\rho$ & $\alpha_{r r}$ & $\alpha_{r b}$ & $\alpha b b$ & $\alpha b r$ \\
\hline Baseline (sessions 1-4) & 8 & 8 & 0 & 0.5625 & 0.7 & 0.3 & 0.7 & 0.3 \\
LowInt (sessions 5-8) & 8 & 8 & 0 & 0.1875 & 0.9 & 0.1 & 0.9 & 0.1 \\
AsySize (sessions 9-12) & 12 & 4 & 0 & 0.375 & 0.9 & 0.1 & 0.7 & 0.3 \\
Govt (sessions 13-16) & 8 & 8 & 0.5 & 0.5625 & $0.7(0.3,0.4)$ & $0.3(0.15,0.15)$ & $0.7(0.3,0.4)$ & $0.3(0.15,0.15)$ \\
\hline \hline
\end{tabular}

Notes. (1) One session of the LowInt treatment has 12 subjects. (2) For the Govt treatment, the two numbers in the brackets represent the probability of meeting a human and a robot, respectively. 
Table 4: Aggregate Token Rejection Rates, \%

\begin{tabular}{crrr|rrr|rrr}
\hline \hline Session & Home-Token Rejection Rate & Foreign-Token Rejection Rate & \multicolumn{3}{|c}{ Home Bias } \\
\hline 1 & $1-75$ & $76-150$ & $1-150$ & $1-75$ & $76-150$ & $1-150$ & $1-75$ & $76-150$ & $1-150$ \\
2 & 4.2 & 0.7 & 2.6 & 18.6 & 7.6 & 12.9 & 14.4 & 6.9 & 10.2 \\
3 & 9.7 & 2.6 & 5.6 & 12.1 & 4.4 & 8.7 & 2.5 & 1.8 & 3.1 \\
4 & 2.2 & 0.0 & 1.1 & 3.3 & 2.9 & 3.1 & 1.1 & 2.9 & 2.0 \\
Baseline Average & 5.2 & 0.8 & 3.0 & 3.1 & 1.2 & 2.2 & -2.1 & 0.4 & -0.8 \\
\hline 5 & $\mathbf{5 . 3}$ & $\mathbf{1 . 0}$ & $\mathbf{3 . 1}$ & $\mathbf{9 . 3}$ & $\mathbf{4 . 0}$ & $\mathbf{6 . 7}$ & $\mathbf{4 . 0}$ & $\mathbf{3 . 0}$ & $\mathbf{3 . 6}$ \\
6 & 2.6 & 0.5 & 1.7 & 20.8 & 9.3 & 15.0 & 18.2 & 8.7 & 13.3 \\
7 & 9.5 & 5.8 & 7.6 & 14.4 & 3.0 & 8.4 & 4.9 & -2.8 & 0.8 \\
8 & 6.3 & 3.4 & 5.2 & 2.2 & 5.0 & 3.8 & -4.1 & 1.6 & -1.3 \\
LowInt Average & 14.0 & 0.0 & 7.7 & 28.4 & 8.5 & 16.8 & 14.5 & 8.5 & 9.1 \\
\hline 9 & $\mathbf{8 . 1}$ & $\mathbf{2 . 4}$ & $\mathbf{5 . 5}$ & $\mathbf{1 6 . 5}$ & $\mathbf{6 . 4}$ & $\mathbf{1 1 . 0}$ & $\mathbf{8 . 4}$ & $\mathbf{4 . 0}$ & $\mathbf{5 . 5}$ \\
10 & 3.0 & 1.1 & 2.3 & 23.4 & 14.9 & 18.0 & 20.4 & 13.8 & 15.8 \\
11 & 8.7 & 0.9 & 4.9 & 6.7 & 5.0 & 5.8 & -2.0 & 4.0 & 0.8 \\
12 & 6.0 & 0.0 & 3.0 & 19.2 & 9.2 & 14.2 & 13.2 & 9.2 & 11.3 \\
AsySize Average & 16.9 & 18.8 & 17.8 & 21.3 & 14.4 & 17.9 & 4.5 & -4.3 & 0.1 \\
\hline 13 & $\mathbf{8 . 7}$ & $\mathbf{5 . 2}$ & $\mathbf{7 . 0}$ & $\mathbf{1 7 . 7}$ & $\mathbf{1 0 . 9}$ & $\mathbf{1 4 . 0}$ & $\mathbf{9 . 0}$ & $\mathbf{5 . 7}$ & $\mathbf{7 . 0}$ \\
14 & 6.4 & 4.2 & 5.4 & 70.0 & 67.2 & 68.3 & 63.6 & 63.0 & 63.0 \\
15 & 7.6 & 1.9 & 4.5 & 54.3 & 54.5 & 54.4 & 46.7 & 52.7 & 49.9 \\
16 & 9.8 & 1.0 & 5.5 & 60.6 & 27.5 & 46.2 & 50.8 & 26.4 & 40.7 \\
Govt Average & 4.0 & 0.9 & 2.4 & 53.4 & 70.7 & 60.6 & 49.4 & 69.8 & 58.2 \\
\hline \hline
\end{tabular}

Notes. (1) For the Govt treatment, only the decisions by human subjects are counted. 
Table 5: Token Rejection Rates by Group, \%

\begin{tabular}{|c|c|c|c|c|c|c|c|c|c|c|c|c|c|c|c|c|c|c|}
\hline \multirow[t]{3}{*}{ Session } & \multicolumn{6}{|c|}{ Home-Token Rejection Rate } & \multicolumn{6}{|c|}{ Foreign-Token Rejection Rate } & \multicolumn{6}{|c|}{ Home Bias } \\
\hline & \multicolumn{3}{|c|}{ Red } & \multicolumn{3}{|c|}{ Blue } & \multicolumn{3}{|c|}{ Red } & \multicolumn{3}{|c|}{ Blue } & \multicolumn{3}{|c|}{ Red } & \multicolumn{3}{|c|}{ Blue } \\
\hline & $1-75$ & $76-150$ & $1-150$ & $1-75$ & $76-150$ & $1-150$ & $1-75$ & $76-150$ & $1-150$ & $1-75$ & $76-150$ & $1-150$ & $1-75$ & $76-150$ & $1-150$ & $1-75$ & $76-150$ & $1-150$ \\
\hline 2 & 10.0 & 5.1 & 7.1 & 9.3 & 0.0 & 4.1 & 21.8 & 5.9 & 14.8 & 2.3 & 2.9 & 2.6 & 11.8 & 0.8 & 7.7 & -7.0 & 2.9 & -1.6 \\
\hline 3 & 1.2 & 0.0 & 0.6 & 3.2 & 0.0 & 1.7 & 1.2 & 0.0 & 0.6 & 5.9 & 5.5 & 5.7 & 0.0 & 0.0 & 0.0 & 2.7 & 5.5 & 4.0 \\
\hline 4 & 8.1 & 1.5 & 4.7 & 2.7 & 0.0 & 1.4 & 3.9 & 1.2 & 2.7 & 2.2 & 1.1 & 1.7 & -4.2 & -0.3 & -2.0 & -0.6 & 1.1 & 0.2 \\
\hline 6 & 0.0 & 3.0 & 1.5 & 20.0 & 8.5 & 13.7 & 0.0 & 0.0 & 0.0 & 29.3 & 6.3 & 17.4 & 0.0 & -3.0 & -1.5 & 9.3 & -2.1 & 3.6 \\
\hline 7 & 4.1 & 0.0 & 2.6 & 8.5 & 6.7 & 7.8 & 0.0 & 1.0 & 0.6 & 4.9 & 8.7 & 7.3 & -4.1 & 1.0 & -2.0 & -3.6 & 2.1 & -0.5 \\
\hline 8 & 20.8 & 0.0 & 11.6 & 7.3 & 0.0 & 4.0 & 24.1 & 14.5 & 18.7 & 33.3 & 2.6 & 14.8 & 3.4 & 14.5 & 7.1 & 26.0 & 2.6 & 10.8 \\
\hline LowInt Average & 6.2 & 0.8 & 3.9 & 10.4 & 4.0 & 7.3 & 10.0 & 4.3 & 6.9 & 23.1 & 9.0 & 15.3 & 3.8 & 3.6 & 3.0 & 12.7 & 4.9 & 8.1 \\
\hline 9 & 1.8 & 0.6 & 1.3 & 8.3 & 12.5 & 8.9 & 30.2 & 19.4 & 23.8 & 14.7 & 11.1 & 12.3 & 28.4 & 18.8 & 22.5 & 6.4 & -1.4 & 3.3 \\
\hline 10 & 10.5 & 1.0 & 5.7 & 0.0 & 0.0 & 0.0 & 7.1 & 10.0 & 8.7 & 6.3 & 0.0 & 3.0 & -3.3 & 9.0 & 3.0 & 6.3 & 0.0 & 3.0 \\
\hline 14 & 11.4 & 3.9 & 7.4 & 4.2 & 0.0 & 1.9 & 42.1 & 39.1 & 40.5 & 63.0 & 71.4 & 66.7 & 30.7 & 35.2 & 33.1 & 58.8 & 71.4 & 64.8 \\
\hline 15 & 2.0 & 0.0 & 1.0 & 17.0 & 2.2 & 10.1 & 78.4 & 44.4 & 64.1 & 37.9 & 8.3 & 24.5 & 76.3 & 44.4 & 63.1 & 20.9 & 6.2 & 14.4 \\
\hline 16 & 4.3 & 0.0 & 2.1 & 3.8 & 1.7 & 2.7 & 47.2 & 70.0 & 55.4 & 63.6 & 71.4 & 67.4 & 43.0 & 70.0 & 53.3 & 59.9 & 69.7 & 64.7 \\
\hline Govt Average & 4.4 & 1.0 & 2.6 & 9.5 & 2.9 & 6.3 & 60.7 & 54.8 & 57.2 & 57.4 & 55.1 & 56.5 & 56.3 & 53.8 & 54.6 & 47.9 & 52.2 & 50.2 \\
\hline
\end{tabular}

Notes. (1) For the Govt treatment, only the decisions by human subjects are counted. 
Table 6: Variables in Probit Regressions

\begin{tabular}{lll}
\hline \hline Dependent Variable & Reject & =0 if accept token for corn; =1 if reject \\
\hline Independent Variable & Period & to capture time trend; value from 1 to 150 \\
& Foreign & dummy: $=1$ if foreign token-corn meeting \\
& Group & dummy: $=0$ if Red group; =1 if Blue group \\
& LowInt & dummy: $=1$ for treatment with low level of integration; =0 otherwise \\
& AsySize & dummy: $=1$ for treatment with asymmetric country sizes; $=0$ otherwise \\
& Govt & dummy: $=1$ for treatment with government agents; =0 otherwise \\
\hline \hline
\end{tabular}


Table 7: Probit Regression-Home Token Rejection



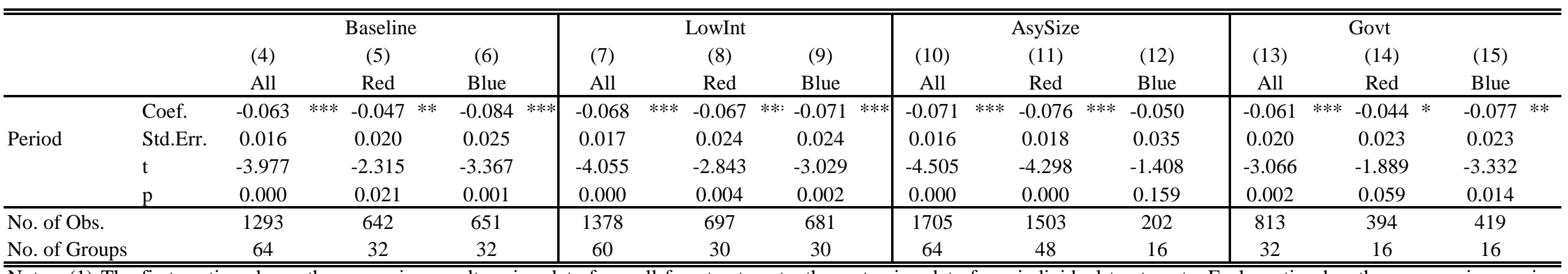

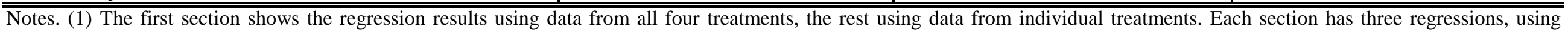
observations on all subjects, subjects in the Red group, and subjects in the Blue group, respectively. (2) The coefficients represent the marginal effects at the mean on the probability of token rejection. (3) ${ }^{*}$ p-value $<=0.1 ; * *$ p-value $<=0.05 ; * * *$ p-value $<=0.01$. 
Table 8: Probit Regression-Foreign Token Rejection

\begin{tabular}{|c|c|c|c|c|c|c|c|}
\hline & & \multicolumn{6}{|c|}{ All Treatments } \\
\hline & & (1) & & (2) & & (3) & \\
\hline & & All & & Red & & Blue & \\
\hline & Coef. & -0.065 & $* * *$ & -0.059 & $* * *$ & -0.069 & $* * *$ \\
\hline \multirow[t]{4}{*}{ Period } & Std.Err. & 0.011 & & 0.014 & & 0.017 & \\
\hline & $\mathrm{t}$ & -5.870 & & -4.221 & & -4.081 & \\
\hline & $\mathrm{p}$ & 0.000 & & 0.000 & & 0.000 & \\
\hline & Coef. & 4.059 & & 0.761 & & 6.704 & $*$ \\
\hline \multirow[t]{4}{*}{ LowInt } & Std.Err. & 3.130 & & 4.171 & & 4.039 & \\
\hline & $\mathrm{t}$ & 1.297 & & 0.182 & & 1.660 & \\
\hline & $\mathrm{p}$ & 0.195 & & 0.855 & & 0.097 & \\
\hline & Coef. & 6.922 & $* *$ & 9.053 & $* *$ & 1.185 & \\
\hline \multirow[t]{4}{*}{ AsySize } & Std.Err. & 3.169 & & 3.775 & & 4.794 & \\
\hline & $\mathrm{t}$ & 2.184 & & 2.398 & & 0.247 & \\
\hline & $\mathrm{p}$ & 0.029 & & 0.016 & & 0.805 & \\
\hline & Coef. & 29.764 & $* * *$ & 30.436 & $* * *$ & 28.201 & $* * *$ \\
\hline \multirow[t]{3}{*}{ Govt } & Std.Err. & 3.377 & & 4.595 & & 4.347 & \\
\hline & $\mathrm{t}$ & 8.813 & & 6.624 & & 6.488 & \\
\hline & $\mathrm{p}$ & 0.000 & & 0.000 & & 0 & \\
\hline No. of Obs. & & 3591 & & 1813 & & 1778 & \\
\hline No. of Groups & & 220 & & 126 & & 94 & \\
\hline
\end{tabular}

\begin{tabular}{|c|c|c|c|c|c|c|c|c|c|c|c|c|c|c|}
\hline & & \multicolumn{4}{|c|}{ Baseline } & \multicolumn{3}{|c|}{ "LowInt } & \multicolumn{3}{|c|}{ "AsySize } & \multicolumn{3}{|c|}{ "Govt } \\
\hline & & (4) & (5) & & (6) & (7) & (8) & (9) & (10) & (11) & (12) & (13) & (14) & (15) \\
\hline & & All & Red & & Blue & All & Red & Blue & All & Red & Blue & All & Red & Blue \\
\hline \multirow{4}{*}{ Period } & Coef. & $-0.062 * * *$ & -0.068 & *** & $-0.053 \quad * *$ & $-0.119 * * *$ & $-0.112 * * *$ & $-0.141^{* * *}$ & $-0.047 * *$ & -0.036 & $-0.049 *$ & 0.022 & 0.012 & 0.032 \\
\hline & Std.Err. & 0.017 & 0.024 & & 0.025 & 0.024 & 0.030 & 0.038 & 0.021 & 0.025 & 0.029 & 0.048 & 0.063 & 0.073 \\
\hline & $\mathrm{t}$ & -3.653 & -2.845 & & -2.166 & -4.959 & -3.681 & -3.746 & -2.283 & -1.406 & -1.679 & 0.458 & 0.187 & 0.441 \\
\hline & $\mathrm{p}$ & 0.000 & 0.004 & & 0.030 & 0.000 & 0.000 & 0.000 & 0.022 & 0.160 & 0.093 & 0.647 & 0.852 & 0.660 \\
\hline No. of Obs. & & 1299 & 646 & & 653 & 1044 & 535 & 509 & 841 & 415 & 426 & 407 & 217 & 190 \\
\hline No. of Groups & & 64 & 32 & & 32 & 60 & 30 & 30 & 64 & 48 & 16 & 32 & 16 & 16 \\
\hline
\end{tabular}

Notes. (1) The first section shows the regression results using data from all four treatments, the rest using data from individual treatments. Each section has three regressions, using observations on all subjects, subjects in the Red group, and subjects in the Blue group, respectively. (2) The coefficients represent the marginal effects at the mean on the probability of token rejection. (3) *p-value $<=0.1 ; * *$ p-value $<=0.05 ; * * *$ p-value $<=0.01$. 
Table 9: Probit Regression-Home Bias (All Treatments)

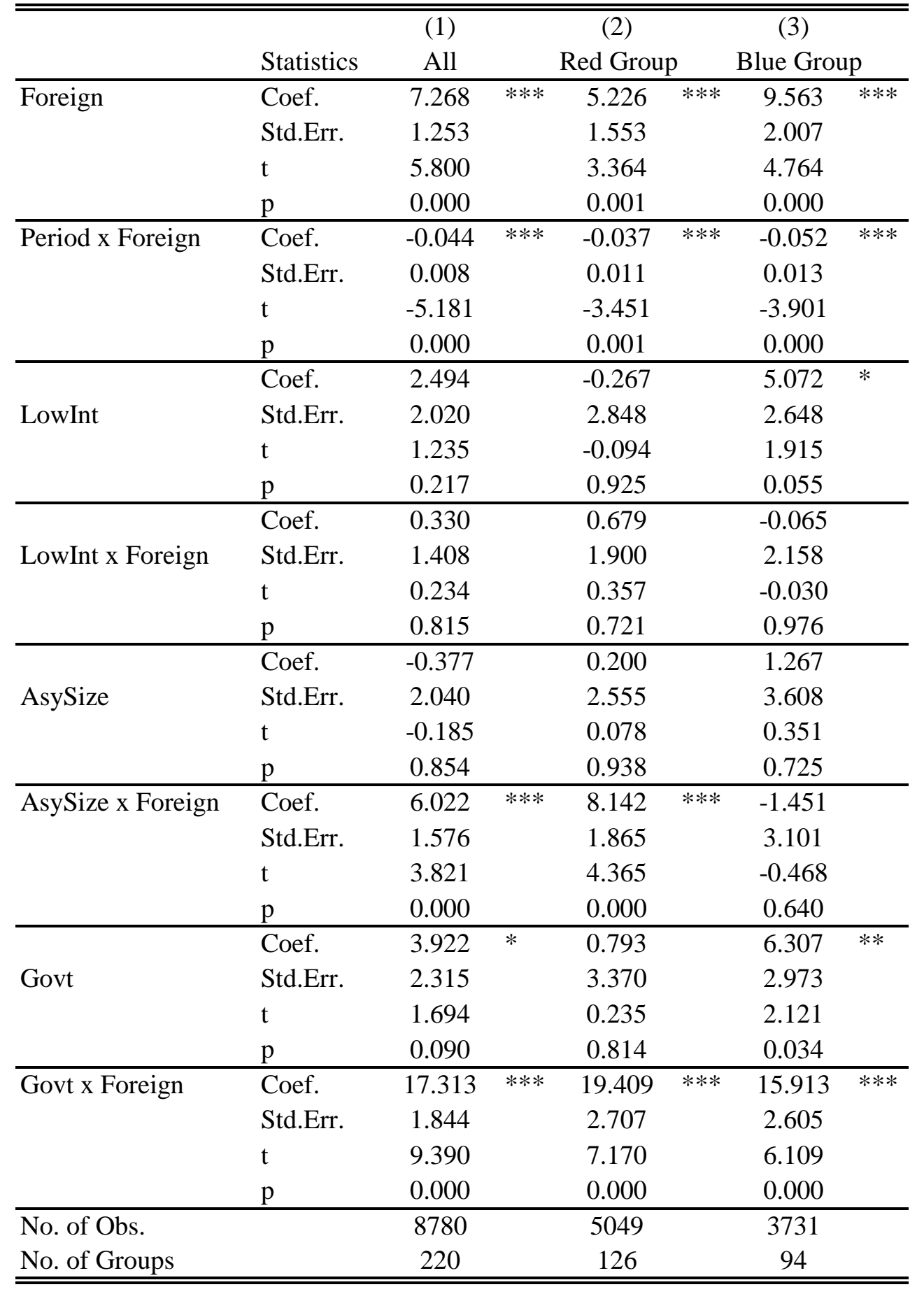

continued ... 
Table 9: Probit Regression-Home Bias (Individual Treatments)

\begin{tabular}{|c|c|c|c|c|c|c|c|c|c|c|c|}
\hline & & \multicolumn{6}{|c|}{ Baseline } & \multicolumn{4}{|c|}{ LowInt } \\
\hline & & (4) & & (5) & & (6) & & (7) & (8) & & (9) \\
\hline & & All & & Red & & Blue & & All & Red & & Blue \\
\hline \multirow[t]{4}{*}{ Foreign } & Coef. & 6.205 & $* * *$ & 5.810 & $* * *$ & 6.476 & $* * *$ & $10.276 * * *$ & 6.006 & $* * *$ & $14.805 * * *$ \\
\hline & Std.Err. & 1.406 & & 1.905 & & 2.048 & & 1.975 & 2.234 & & 3.142 \\
\hline & $\mathrm{t}$ & 4.413 & & 3.049 & & 3.162 & & 5.202 & 2.689 & & 4.711 \\
\hline & $\mathrm{p}$ & 0.000 & & 0.002 & & 0.002 & & 0.000 & 0.007 & & 0.000 \\
\hline \multirow[t]{4}{*}{ Period x Foreign } & Coef. & -0.044 & $* * *$ & -0.057 & $* * *$ & -0.032 & * & $-0.082 * * *$ & -0.048 & $* *$ & $-0.117^{* * *}$ \\
\hline & Std.Err. & 0.013 & & 0.020 & & 0.018 & & 0.019 & 0.023 & & 0.031 \\
\hline & $\mathrm{t}$ & -3.318 & & -2.787 & & -1.751 & & -4.207 & -2.142 & & -3.755 \\
\hline & $\mathrm{p}$ & 0.001 & & 0.005 & & 0.080 & & 0.000 & 0.032 & & 0.000 \\
\hline No. of Obs. & & 2592 & & 1288 & & 1304 & & 2422 & 1232 & & 1190 \\
\hline No. of Groups & & 64 & & 32 & & 32 & & 60 & 30 & & 30 \\
\hline
\end{tabular}

\begin{tabular}{|c|c|c|c|c|c|c|c|c|}
\hline & & \multicolumn{4}{|c|}{ AsySize } & \multicolumn{3}{|c|}{ Govt } \\
\hline & & (10) & & (11) & (12) & (13) & (14) & (15) \\
\hline & & All & & Red & Blue & All & Red & Blue \\
\hline \multirow[t]{4}{*}{ Foreign } & Coef. & 12.548 & $* * *$ & $13.560 * * *$ & $6.623 * *$ & $34.567 * * *$ & $35.914 * * *$ & $33.721 * * *$ \\
\hline & Std.Err. & 2.153 & & 2.639 & 3.235 & 2.669 & 3.452 & 3.971 \\
\hline & $\mathrm{t}$ & 5.829 & & 5.139 & 2.047 & 12.949 & 10.405 & 8.491 \\
\hline & $\mathrm{p}$ & 0.000 & & 0.000 & 0.041 & 0.000 & 0.000 & 0.000 \\
\hline \multirow[t]{4}{*}{ Period x Foreign } & Coef. & -0.037 & $* *$ & -0.030 & $-0.043 *$ & 0.011 & 0.009 & 0.009 \\
\hline & Std.Err. & 0.016 & & 0.020 & 0.026 & 0.027 & 0.034 & 0.044 \\
\hline & $\mathrm{t}$ & -2.306 & & -1.479 & -1.651 & 0.401 & 0.271 & 0.201 \\
\hline & $\mathrm{p}$ & 0.021 & & 0.139 & 0.099 & 0.689 & 0.786 & 0.840 \\
\hline No. of Obs. & & 2546 & & 1918 & 628 & 1220 & 611 & 609 \\
\hline No. of Groups & & 64 & & 48 & 16 & 32 & 16 & 16 \\
\hline
\end{tabular}

Notes. (1) The first section shows the regression results using data from all four treatments, the rest using data from individual treatments. Each section has three regressions, using observations on all subjects, subjects in the Red group, and subjects in the Blue group, respectively. (2) The coefficients represent the marginal effects at the mean on the probability of token rejection. (3) ${ }^{*} \mathrm{p}$-value $<=0.1$; ${ }^{* *} \mathrm{p}$-value $<=0.05$; *** $\mathrm{p}$-value $<=0.01$. 
Table 10: Distribution of Assets, \%

\begin{tabular}{ccccccc}
\hline \hline Session & $\mathrm{Rr}$ & $\mathrm{Rb}$ & $\mathrm{R} 0$ & $\mathrm{Br}$ & $\mathrm{Bb}$ & $\mathrm{B} 0$ \\
\hline 1 & 28.2 & 22.7 & 49.2 & 21.8 & 27.3 & 50.8 \\
2 & 26.3 & 22.4 & 51.2 & 23.7 & 27.6 & 48.8 \\
3 & 26.3 & 22.8 & 51.0 & 23.8 & 27.3 & 49.0 \\
4 & 20.4 & 28.2 & 51.4 & 29.6 & 21.8 & 48.6 \\
Baseline Average & 25.3 & 24.0 & 50.7 & 24.7 & 26.0 & 49.3 \\
Simulated Average & 25.9 & 24.0 & 50.1 & 24.1 & 26.0 & 49.9 \\
\hline 5 & 35.1 & 16.1 & 48.8 & 14.9 & 33.9 & 51.2 \\
6 & 25.3 & 24.4 & 50.2 & 24.7 & 25.6 & 49.8 \\
7 & 24.8 & 25.4 & 49.8 & 25.3 & 24.6 & 50.2 \\
8 & 31.3 & 19.4 & 49.3 & 18.7 & 30.6 & 50.7 \\
LowInt Average & 29.1 & 21.3 & 49.5 & 20.9 & 28.7 & 50.5 \\
Simulated Average & 26.8 & 23.6 & 49.6 & 23.2 & 26.4 & 50.4 \\
\hline 9 & 39.7 & 11.1 & 49.3 & 31.0 & 16.8 & 52.2 \\
10 & 39.2 & 9.4 & 51.4 & 32.5 & 21.7 & 45.8 \\
12 & 35.3 & 13.2 & 51.4 & 44.0 & 10.3 & 45.7 \\
AsySize Average & 40.0 & 8.9 & 51.1 & 30.0 & 23.3 & 46.7 \\
Simulated Average & 38.5 & 10.7 & 50.8 & 34.4 & 18.0 & 47.6 \\
\hline \hline
\end{tabular}

Notes. (1) Rr, Rb and R0 (Br, Bb and B0) represent the percentage of agents in the Red (Blue) group holding the red token, the blue token, and corn, respectively. (2) The numbers are calculated as the average across all 150 rounds in each session. 


\section{Appendix A: Model with Government Transaction Policies}

In this Appendix, we generalize the two-group, two-currency model to include an additional type of agent, called government agents, who follow exogenous trading strategies, as in Aiyagari and Wallace (1997) and Li and Wright (1998). We characterize the conditions under which each type of currency regime emerges as an equilibrium.

There is a constant fraction $g_{r} \in[0,1]$ of government agents in the Red group and a constant fraction $g_{b} \in[0,1]$ of government agents in the Blue group. As a result, there are $g_{i} n_{i}$ government agents in group $i$ and $\left(1-g_{i}\right) n_{i}$ private agents in group $i$.

Government agents consume and produce like private agents and are subject to the same matching process. However in contrast with private agents who adopt trading strategies based on maximizing behavior, government agents follow exogenous trading rules called government transaction policies. In what follows, we consider a benchmark policy where government agents only accept domestic currency in exchange for goods (when they are sellers), and always accept goods for any currency (when they are buyers).

We use the vector $\mathbf{m}=\left(m_{i k} ; m_{i}^{g}\right)_{i, k \in\{r, b\}}$ to summarize the distribution of currencies in the economy, where $m_{i k}$ denotes the fraction of private buyers from group $i$ with currency $k$, and $m_{i}^{g}$ denotes the fraction of government agents from group $i$ with currency $i$ (given a policy where government agents always reject foreign currency, a government agent from $i$ would never hold currency $j \neq i$ ). We use

$$
m_{i 0}=1-\left(m_{i r}+m_{i b}\right)
$$

to denote the fraction of private sellers in group $i$, and

$$
m_{i 0}^{g}=1-m_{i}^{g},
$$

to denote the fraction of government sellers in group $i$. At any point of time, the aggregate supply of currency $i$ must equal its aggregate demand:

$$
n_{i} M_{i}=n_{i}\left[\left(1-g_{i}\right) m_{i i}+g_{i} m_{i}^{g}\right]+n_{j}\left(1-g_{j}\right) m_{j i} .
$$

We will focus on stationary equilibria where the distribution of assets is constant over time. Given the foreign currency acceptance decision, $\lambda_{i \in\{r, b\}}$, the distribution of assets $\left(m_{i i}, m_{i j}, m_{i 0}, m_{i}^{g}, m_{i 0}^{g}\right)_{i \in\{r, b\}, j \neq i}$ satisfies

$$
\begin{aligned}
m_{i 0}\left[\alpha_{i i} g_{i} m_{i}^{g}+\alpha_{i j}\left(1-g_{j}\right) m_{j i}\right] & =m_{i i}\left[\alpha_{i i} g_{i} m_{i 0}^{g}+\alpha_{i j}\left(1-g_{j}\right) m_{j 0} \lambda_{j}\right], \\
m_{i 0} \alpha_{i j}\left[\left(1-g_{j}\right) m_{j j}+g_{j} m_{j}^{g}\right] & =m_{i j} \alpha_{i j}\left[\left(1-g_{j}\right) m_{j 0}+g_{j} m_{j 0}^{g}\right] \rightarrow \\
m_{i 0}\left[\left(1-g_{j}\right) m_{j j}+g_{j} m_{j}^{g}\right] & =m_{i j}\left[\left(1-g_{j}\right) m_{j 0}+g_{j} m_{j 0}^{g}\right], \\
m_{i 0}^{g}\left[\alpha_{i i}\left(1-g_{i}\right) m_{i i}+\alpha_{i j}\left(1-g_{j}\right) m_{j i}\right] & =m_{i}^{g}\left[\alpha_{i i}\left(1-g_{i}\right) m_{i 0}+\alpha_{i j}\left(1-g_{j}\right) m_{j 0} \lambda_{j}\right] .
\end{aligned}
$$

Equation (4) states that the reduction in $m_{i i}$ (RHS) equals the increase in $m_{i i}$ (LHS). When a group $i$ buyer holding currency $i$ purchases a good from a local government seller or a foreign private seller who accepts currency $i$ (remember that a government agent $j$ does not accept currency $r$ ), $m_{i i}$ will decrease (note that we do not need to consider the case where the agent meets a local private agent since the transaction involves agents belonging to the same group). Similarly, (5) describes the changes in $m_{i j}$ : the RHS the decrease in $m_{i j}$ (when a group $i$ buyer with currency $b$ uses it to buy a good from foreign private and government agents), and the LHS the increase in $m_{i j}$ (when a group $i$ seller acquires currency $b$ from foreign private and government agents). Finally, (6) balances the changes in $m_{i}^{g}$ : the RHS captures the decrease in $m_{i}^{g}$ (when a group $i$ government buyer purchases a good from a home private seller or a foreign private buyer who accepts currency $i$ ), and the LHS captures the increase in $m_{i}^{g}$ (as a group $i$ seller acquires currency $i$ from home or foreign private buyers).

As before, let $V_{i 0}$ denote the lifetime utility of a seller from group $i$ who holds no money and $V_{i k}$ denote the lifetime utility of an agent from group $i$ who starts a period with currency $k$. The flow value of being a seller from group $i$ can be written as

$$
\begin{aligned}
r V_{i 0}= & \left\{\alpha_{i i}\left[\left(1-g_{i}\right) m_{i i}+g_{i} m_{i}^{g}\right]+\alpha_{i j}\left(1-g_{j}\right) m_{j i}\right\}\left(V_{i i}-V_{i 0}\right) \\
& +\left\{\alpha_{i i}\left(1-g_{i}\right) m_{i j}+\alpha_{i j}\left[\left(1-g_{j}\right) m_{j j}+g_{j} m_{j}^{g}\right]\right\} \lambda_{i}\left(V_{i j}-V_{i 0}\right),
\end{aligned}
$$

which consists of the probability he/she meets a local or foreign buyer holding currency $i$ times the trade surplus in that meeting, plus the probability he/she meets a local or foreign buyer holding currency $j$. 
The flow value of being a buyer from $i$ holding domestic currency is

$$
r V_{i i}=\left\{\alpha_{i i}\left[\left(1-g_{i}\right) m_{i 0}+g_{i} m_{i 0}^{g}\right]+\alpha_{i j}\left(1-g_{j}\right) \lambda_{j} m_{j 0}\right\}\left(u+V_{i 0}-V_{i i}\right),
$$

which consists of the probability the buyer meets a domestic or foreign seller times the gains from trading. Notice a buyer from $i$ can be in one type of domestic meeting and two types of foreign meetings: with probability $\alpha_{i i}$, the buyer meets a local seller (either private or government) who always accepts domestic currency; with probability $\alpha_{i j}\left(1-g_{j}\right)$, the buyer meets a foreign private seller who follows an endogenous trading strategy $\lambda_{i}$, and with probability $\alpha_{i j} g_{j}$, the buyer meets a foreign government seller who always rejects the buyer's currency.

Similarly, the flow value of being a buyer from $i$ holding foreign currency is

$$
r V_{i j}=\left[\alpha_{i i}\left(1-g_{i}\right) \lambda_{i} m_{i 0}+\alpha_{i j}\left\{\left(1-g_{j}\right) m_{j 0}+g_{j} m_{j 0}^{g}\right]\right\}\left(u+V_{i 0}-V_{i j}\right) .
$$

\section{Regime N: Two National Currencies}

In this equilibrium, private and government agents adopt the same strategy and do not accept foreign currency. The asset distribution satisfies

$$
\begin{aligned}
m_{i i} & =m_{i}^{g}=M_{i}, \\
m_{i j} & =0 \\
m_{i 0} & =m_{i 0}^{g}=1-M_{i} .
\end{aligned}
$$

The flow Bellman equations are

$$
\begin{gathered}
r V_{i 0}=\alpha_{i i} M_{i}\left(V_{i i}-V_{i 0}\right), \\
r V_{i i}=\alpha_{i i}\left(1-M_{i}\right)\left(u+V_{i 0}-V_{i i}\right), \\
r V_{i j}=\alpha_{i j}\left(1-M_{j}\right)\left(u+V_{i 0}-V_{i j}\right) .
\end{gathered}
$$

The seller's incentive condition to never accept foreign currency, i.e., $V_{i j} \leq V_{i 0}$, is satisfied if

$$
\alpha_{i j} \leq \frac{\alpha_{i i}^{2} M_{i}\left(1-M_{i}\right)}{\left(r+\alpha_{i i}\right)\left(1-M_{j}\right)},
$$

which is the same existence condition as in the baseline model without government agents. Note that we can show that, in this equilibrium, $V_{i i}>V_{i 0}$, which guarantees that national currencies are always accepted.

\section{Regime $R$ or $B$ : One International Currency}

The emergence of an international currency occurs when private sellers from one group accept both currencies. Here we focus on the case where private sellers from Blue accept both currencies, while private sellers from Red accept only domestic money, i.e., $\lambda=(0,1)$. We call this Regime $R$. In this regime, the red token emerges as the sole international medium of exchange. Because Red private and government agents adopt the same strategy, they have the same asset distribution and we can treat them as a single group:

$$
\begin{aligned}
& m_{r b}=0, \\
& m_{r r}=m_{r}^{g}=M_{r}-\frac{n_{b}\left(1-g_{b}\right)}{n_{r}} m_{b r}, \\
& m_{r 0}=m_{r 0}^{g}=1-m_{r r},
\end{aligned}
$$

where the second equation utilizes the market-clearing condition for red tokens. From the above three equations, we can acquire the Red agents' asset distribution once we know $m_{b r}$.

The stationary equilibrium condition for $m_{r r}\left(\right.$ and $\left.m_{r}^{g}\right)$ is $m_{r 0} \alpha_{r b}\left(1-g_{b}\right)=m_{r r} \alpha_{r b}\left(1-g_{b}\right) m_{b r}$ or

$$
m_{r r} m_{b 0}=m_{r 0} m_{b r} \text {. }
$$

Note that the condition is also equivalent to the stationary equilibrium condition for $m_{b r}$ because red tokens only flow between Red agents (private and government as a whole) and private Blue agents. Using the market-clearing condition for blue tokens, $\left(1-g_{b}\right) m_{b b}+g_{b} m_{b}^{g}=M_{b}$, we have

$$
m_{b}^{g}=\frac{M_{b}-\left(1-g_{b}\right) m_{b b}}{g_{b}} .
$$


The stationary equilibrium conditions for $m_{b b}$ is

$$
m_{b 0} m_{b}^{g}=m_{b b} m_{b 0}^{g} .
$$

Combining equations (11) to (13) with $m_{b 0}=1-m_{b b}-m_{b r}$ and $m_{b 0}^{g}=1-m_{b}^{g}$, we can solve the Blue agents' asset distribution $\left(m_{b r}, m_{b b}, m_{b 0}, m_{b}^{g}, m_{b 0}^{g}\right)$. After that, we can solve the Red agents' asset distribution.

After solving the stationary asset distribution, we check the conditions for the strategy $\lambda=(0,1)$. For Red agents, the value functions are

$$
\begin{aligned}
& r V_{r 0}=\underbrace{\left[\alpha_{r r} m_{r r}+\alpha_{r b}\left(1-g_{b}\right) m_{b r}\right]}_{A_{0}}\left(V_{r r}-V_{r 0}\right), \\
& r V_{r r}=\underbrace{\left[\alpha_{r r} m_{r 0}+\alpha_{r b}\left(1-g_{b}\right) m_{b 0}\right]}_{A_{r}}\left(u+V_{r 0}-V_{r r}\right), \\
& r V_{r b}=\underbrace{\alpha_{r b}\left[\left(1-g_{b}\right) m_{b 0}+g_{b} m_{b 0}^{g}\right]}_{A_{b}}\left(u+V_{r 0}-V_{r b}\right) .
\end{aligned}
$$

For Blue agents, the value functions are

$$
\begin{aligned}
r V_{b 0}= & \underbrace{\alpha_{b b}\left[\left(1-g_{b}\right) m_{b b}+g_{b} m_{b}^{g}\right]}_{B_{0 b}}\left(V_{b b}-V_{b 0}\right) \\
& +\underbrace{\left[\alpha_{b b}\left(1-g_{b}\right) m_{b r}+\alpha_{b r} m_{r r}\right]}_{B_{0 r}}\left(V_{b r}-V_{b 0}\right), \\
r V_{b b}= & \underbrace{\alpha_{b b}\left[\left(1-g_{b}\right) m_{b 0}+g_{b} m_{b 0}^{g}\right]}_{B_{b}}\left(u+V_{b 0}-V_{b b}\right), \\
r V_{b r}= & \underbrace{\left[\alpha_{b b}\left(1-g_{b}\right) m_{b 0}+\alpha_{b r} m_{r 0}\right]}_{B r}\left(u+V_{b 0}-V_{b r}\right) .
\end{aligned}
$$

It can be shown that in this equilibrium, $V_{r r}>V_{r 0}$, which guarantees sellers from Red accept the red token. To ensure that sellers from Blue accept the red token, i.e., $V_{b r}>V_{b 0}$, we need

$$
B_{r}\left(r+B_{0 b}+B_{b}\right)>B_{0 b} B_{b} .
$$

This is different from the case without government agents (if $g=0$, then $V_{b r}>V_{b 0}$ is automatically satisfied). To ensure that sellers from Red will not accept the blue token, i.e., $V_{r b} \leq V_{r 0}$, we need

$$
A_{b}\left(r+A_{0}+A_{r}\right)<A_{0} A_{r} .
$$

To ensure private sellers from Blue are willing to accept the blue token, $V_{b b} \geq V_{b 0}$ must hold, which requires

$$
B_{b}\left(r+B_{0 r}+B_{r}\right)>B_{0 r} B_{r}
$$

\section{Regime $U$ : Two International Currencies}

In this equilibrium, all private agents share the same strategy, $\lambda=(1,1)$. As a result, the distribution of assets is the same in the private sectors across the two countries

$$
\begin{aligned}
& m_{r r}=m_{b r}=m_{r}=\frac{n_{r}\left(M_{r}-g_{r} m_{r}^{g}\right)}{n_{r}\left(1-g_{r}\right)+n_{b}\left(1-g_{b}\right)}, \\
& m_{b r}=m_{b b}=m_{b}=\frac{n_{b}\left(M_{b}-g_{b} m_{b}^{g}\right)}{n_{r}\left(1-g_{r}\right)+n_{b}\left(1-g_{b}\right)} .
\end{aligned}
$$

We only need to consider stationary-equilibrium conditions that describe how currencies flow between private agents and group $i$ government agents:

$$
\begin{aligned}
& m_{r}\left[\frac{\alpha_{r r} n_{r}\left(1-g_{r}\right)+\alpha_{r b} n_{b}\left(1-g_{b}\right)}{n_{r}\left(1-g_{r}\right)+n_{b}\left(1-g_{b}\right)}\right]\left(1-g_{r}\right) m_{r 0}^{g}=m_{0}\left[\frac{\alpha_{r r} n_{r}\left(1-g_{r}\right)+\alpha_{r b} n_{b}\left(1-g_{b}\right)}{n_{r}\left(1-g_{r}\right)+n_{b}\left(1-g_{b}\right)}\right]\left(1-g_{r}\right) m_{r}^{g}, \\
& m_{b}\left[\frac{\alpha_{r r} n_{r}\left(1-g_{r}\right)+\alpha_{r b} n_{b}\left(1-g_{b}\right)}{n_{r}\left(1-g_{r}\right)+n_{b}\left(1-g_{b}\right)}\right]\left(1-g_{b}\right) m_{b 0}^{g}=m_{0}\left[\frac{\alpha_{r r} n_{r}\left(1-g_{r}\right)+\alpha_{r b} n_{b}\left(1-g_{b}\right)}{n_{r}\left(1-g_{r}\right)+n_{b}\left(1-g_{b}\right)}\right]\left(1-g_{b}\right) m_{b}^{g},
\end{aligned}
$$


which can be simplified to

$$
\begin{aligned}
& m_{r} m_{r 0}^{g}=m_{0} m_{r}^{g}, \\
& m_{b} m_{b 0}^{g}=m_{0} m_{b}^{g} .
\end{aligned}
$$

Using $m_{r}+m_{b}+m_{0}=1$ and $m_{i}^{g}+m_{i 0}^{g}=1$, we can rewrite the above two equations as

$$
\begin{aligned}
& m_{r}=\left(1-m_{b}\right) m_{r}^{g}, \\
& m_{b}=\left(1-m_{r}\right) m_{b}^{g} .
\end{aligned}
$$

Equations (20)-(23) can be used to solve for the stationary distribution $\left(m_{r}, m_{b}, m_{r}^{g}, m_{b}^{g}\right)$.

The value functions for group $i$ agents are

$$
\begin{aligned}
r V_{i 0}= & \underbrace{\left[\alpha_{i i}\left(1-g_{i}\right) m_{i}+\alpha_{i j}\left(1-g_{j}\right) m_{i}+\alpha_{i i} g_{i} m_{i}^{g}\right]}_{A_{0 i}}\left(V_{i i}-V_{i 0}\right) \\
& +\underbrace{\left[\alpha_{i i}\left(1-g_{i}\right) m_{j}+\alpha_{i j}\left(1-g_{j}\right) m_{j}+\alpha_{i j} g_{j} m_{j}^{g}\right]}_{A_{0 j}}\left(V_{i j}-V_{i 0}\right), \\
r V_{i i}= & \underbrace{\left[\alpha_{i i}\left(1-g_{i}\right) m_{0}+\alpha_{i j}\left(1-g_{j}\right) m_{0}+\alpha_{i i} g_{i} m_{i 0}^{g}\right]}_{A_{i}}\left(u+V_{i 0}-V_{i i}\right), \\
r V_{i j}= & \underbrace{\left[\alpha_{i i}\left(1-g_{i}\right) m_{0}+\alpha_{i j}\left(1-g_{j}\right) m_{0}+\alpha_{i j} g_{j} m_{j 0}^{g}\right]}_{A_{j}}\left(u+V_{i 0}-V_{i j}\right) .
\end{aligned}
$$

To ensure that both currencies are international, we need $V_{i i}>V_{i 0}$ and $V_{i j}>V_{i 0}$. Unlike in the case without government agents, we may have $V_{i i} \neq V_{i j}$ if $\alpha_{i i} g_{i} m_{i 0}^{g} \neq \alpha_{i j} g_{j} m_{j 0}^{g}$. The condition for $V_{i i}>V_{i 0}$ is

$$
\frac{\frac{A_{0 i} A_{i}}{r+A_{i}}+\frac{A_{0 j} A_{j}}{r+A_{j}}}{r+A_{0 i}+A_{0 j}-\frac{A_{0 i} A_{i}}{r+A_{i}}-\frac{A_{0 j} A_{j}}{r+A_{j}}}<\frac{A_{i}}{r} .
$$

The condition for $V_{i j}>V_{i 0}$ is

$$
\frac{\frac{A_{0 i} A_{i}}{r+A_{i}}+\frac{A_{0 j} A_{j}}{r+A_{j}}}{r+A_{0 i}+A_{0 j}-\frac{A_{0 i} A_{i}}{r+A_{i}}-\frac{A_{0 j} A_{j}}{r+A_{j}}}<\frac{A_{j}}{r} .
$$

In the Govt treatment, we have $n_{r}=n_{b}=n, g_{r}=g_{b}=g$ and $M_{i}=M_{j}=M=1 / 2$. In this case, the stationary asset distribution in Regime $U$ satisfies $m_{r}=m_{b}=m$ and $m_{b}^{g}=m_{r}^{g}=m^{g}$, where $\left(m, m^{g}\right)$ solves

$$
\begin{aligned}
(1-g) m+g m^{g} & =M \rightarrow m^{g}=\frac{M-(1-g) m}{g}, \\
m & =(1-m) m^{g} .
\end{aligned}
$$

The value functions simplify to

$$
\begin{aligned}
& r V_{i 0}=\underbrace{\left[(1-g) m+\alpha_{i i} g m^{g}\right]}_{A_{0 i}}\left(V_{i i}-V_{i 0}\right)+\underbrace{\left[(1-g) m+\alpha_{i j} g m^{g}\right]}_{A_{0 j}}\left(V_{i j}-V_{i 0}\right), \\
& r V_{i i}=\underbrace{\left[(1-g) m_{0}+\alpha_{i i} g m_{0}^{g}\right]}_{A_{i}}\left(u+V_{i 0}-V_{i i}\right), \\
& r V_{i j}=\underbrace{\left[(1-g) m_{0}+\alpha_{i j} g m_{0}^{g}\right]}_{A_{j}}\left(u+V_{i 0}-V_{i j}\right) .
\end{aligned}
$$

Given that $\alpha_{i i}>\alpha_{i j}$, we have $V_{i i}>V_{i j}$, and $V_{i i}>V_{i 0}$ is automatically satisfied. 


\section{Appendix B: Matching Process}

In this Appendix we describe how we implement the matching process in the laboratory. The number of agents in the Red (Blue) group is $n_{r}\left(n_{b}\right)$. Both are multiples of two. Without loss of generality, assume $n_{r} \geq n_{b}$. In each round of trading, the following two-step matching process is implemented.

Step 1. Use Z-tree's "stranger matching" protocol to randomly match subjects into pairs (which is equivalent to assuming full integration). There are three types of pairing: international (RB), Red domestic (RR) and Blue domestic (BB).

Step 2. Partition all RB pairs into groups of two RB pairs and reorganize each group into two domestic pairs (1 $\mathrm{RR}+1 \mathrm{BB})$ with probability $1-\rho$. The parameter $\rho$ controls the level of integration. Full integration is achieved by setting $\rho=1$, and full isolation by setting $\rho=0$.

Next we translate the parameter $\rho$ into meeting probabilities $\alpha_{i j}$ with $i, j=\{r, b\}$. Step 1 of the matching process generates $F\left(n_{r}+n_{b}\right)$ possible pairing profiles, where the function $F(N)$ is defined as

$$
F(N)=(N-1)(N-3) * \ldots * 3 * 1 .
$$

There are $n_{b} / 2+1$ possible matching outcomes in terms of the number of international pairs, denoted by $k \in$ $\left.\left\{0,2, \ldots, n_{b}\right\}\right) .{ }^{1}$ Among the $F\left(n_{r}+n_{b}\right)$ pairing profiles, $D_{k}=C_{n_{r}}^{k} C_{n_{b}}^{k} k ! F\left(n_{r}-k\right) F\left(n_{b}-k\right)$ of them feature outcome $k$. The probability of outcome $k$ occurring following the random matching protocol in Step 1 is therefore $w_{k}=$ $D_{k} / F\left(n_{r}+n_{b}\right)$. We then adjust $w_{k}$ to account for the second step of the matching process. The magnitude of adjustment $\Delta_{k}$ is listed in Table B1. We denote the post-adjustment probability $W_{k}$.

We will illustrate how to derive the probability of outcome $k=0$. Following Step 1 , the probability that outcome 0 occurs is $w_{0}=F\left(n_{r}\right) F\left(n_{b}\right) / F\left(n_{r}+n_{b}\right)$. To adjust for Step 2, note that all Step 1 pairing profiles with $k=2$ become $k=0$ with probability $(1-\rho)$, and all Step 1 pairing profiles with $k=4$ become $k=0$ with probability $(1-\rho)^{2}$, and so on. As a result, we augment $w_{0}$ by $\Delta_{0}=w_{2}(1-\rho)+w_{4}(1-\rho)^{2}+w_{6}(1-\rho)^{3}+\ldots+w_{n_{b}}(1-\rho)^{n_{b} / 2}$ to derive $W_{0}$, the probability of outcome $k=0$ following the two steps of the matching process.

Table B1: Probability of Matching Outcomes

\begin{tabular}{lll}
\hline$k$ & \multicolumn{1}{c}{$D_{k}$} & $\Delta_{2}(1-\rho)+w_{4}(1-\rho)^{2}+w_{6}(1-\rho)^{3}+\ldots+w_{n_{b}}(1-\rho)^{n_{b} / 2}$ \\
\hline 0 & $F\left(n_{r}\right) F\left(n_{b}\right)$ & $-w_{2}(1-\rho)+2 w_{4} \rho(1-\rho)+3 w_{6} \rho(1-\rho)^{2}+\ldots+\frac{n_{b}}{2} w_{n_{b}} \rho(1-\rho)^{n_{b} / 2-1}$ \\
2 & $C_{n_{r}}^{2} C_{n_{b}}^{2} 2 ! F\left(n_{r}-2\right) F\left(n_{b}-2\right)$ & $-w_{4}\left(1-\rho^{2}\right)+3 w_{6} \rho^{2}(1-\rho)+\ldots+C_{n_{b} / 2}^{2} w_{n_{b}} \rho^{2}(1-\rho)^{n_{b} / 2-2}$ \\
4 & $C_{n_{r}}^{4} C_{n_{b}}^{4} 4 ! F\left(n_{r}-4\right) F\left(n_{b}-4\right)$ & $-w_{6}\left(1-\rho^{3}\right)+C_{4}^{3} w_{8} \rho^{3}(1-\rho)+\ldots+C_{n_{b} / 2}^{3} w_{n_{b}} \rho^{3}(1-\rho)^{n_{b} / 2-3}$ \\
6 & $C_{n_{r}}^{6} C_{n_{b}}^{6} 6 ! F\left(n_{r}-6\right) F\left(n_{b}-6\right)$ & $-w^{4}$ \\
8 & $C_{n_{r}}^{8} C_{n_{b}}^{8} 8 ! F\left(n_{r}-8\right) F\left(n_{b}-8\right)$ & $-w_{8}\left(1-\rho^{4}\right)+C_{5}^{4} w_{10} \rho^{4}(1-\rho)+\ldots+C_{n_{b} / 2}^{4} w_{n_{b}} \rho^{4}(1-\rho)^{n_{b} / 2-4}$ \\
& & \\
$k$ & $C_{n_{r}}^{k} C_{n_{b}}^{k} k ! F\left(n_{r}-k\right) F\left(n_{b}-k\right)$ & $-w_{k}\left(1-\rho^{k / 2}\right)+C_{\frac{k}{2}+1}^{\frac{k}{2}} w_{k+2} \rho^{\frac{k}{2}}(1-\rho)+\ldots+C_{n_{b} / 2}^{k / 2} w_{n_{b}} \rho^{\frac{k}{2}}(1-\rho)^{\frac{n_{b}-k}{2}}$ \\
& & \\
$n_{b}$ & $C_{n_{r}}^{n_{b}} n_{b} ! F\left(n_{r}-n_{b}\right)$ & $-w_{n_{b}}\left(1-\rho^{\frac{n_{b}}{2}}\right)$
\end{tabular}

After deriving $W_{k}$, we can calculate the probability of match types $\alpha_{i j}$ in each trading round as follows:

Table B2: Meeting Probabilities

\begin{tabular}{lll}
\hline & Red & Blue \\
\hline Red & $\alpha_{r r}=1-\alpha_{r b}$ & $\alpha_{r b}=\sum_{k=0}^{n_{b}}\left(W_{k} k / n_{r}\right)$ \\
Blue & $\alpha_{b r}=\sum_{k=0}^{n_{b}}\left(W_{k} k / n_{b}\right)$ & $\alpha_{b b}=1-\alpha_{b r}$ \\
\hline
\end{tabular}

\footnotetext{
${ }^{1}$ Each matching outcome is also unique in terms of the number of Red domestic pairs, which is equal to $\left(n_{r}-k\right) / 2$, and the number of Blue domestic pairs, which is equal to $\left(n_{b}-k\right) / 2$.
} 


\section{Appendix C: Equilibria with Finite Population}

Following Duffy and Ochs (2002), we take two steps to verify the existence of each type of equilibrium listed in Table 2 with a finite population. ${ }^{1}$

1. Calculate the expected lifetime utility of an individual seller by following the equilibrium strategy and by following each of all possible deviation strategies, assuming that all other agents follow the equilibrium strategy.

2. Verify that the individual's lifetime utility is maximized by following the equilibrium strategy, or that unilateral deviations from the equilibrium strategy are not profitable.

\section{Regime N: Two National Currencies}

In an equilibrium with two national currencies, the equilibrium strategy is to accept only home currency. Possible deviation strategies are (i) accept both currencies, (ii) accept only the foreign currency, and (iii) accept neither currency. Deviation (iii) is obviously a dominated strategy, so we will consider only deviations (i) and (ii).

We will check the incentives for a Red agent. The analysis is similar for Blue agents (simply swap $r$ and $b$ ). We can represent the equilibrium and deviation strategies in the table below, where "1" stands for accept, and "0" stands for reject.

Table C1: Red Agent's Equilibrium and Deviation Strategies in Regime $N$

\begin{tabular}{cccc}
\hline & Equilibrium & Deviation (i) & Deviation (ii) \\
\hline Token $r$ & 1 & 1 & 0 \\
Token $b$ & 0 & 1 & 1 \\
\hline
\end{tabular}

Equilibrium Strategy - Accept Only Home Currency. If everyone follows the equilibrium strategy, the distribution of assets is described by the table below (for example, the first cell represents the number of tokens $r$ held by Red agents).

Table C2: Asset Distribution in Regime $N$ under Equilibrium Strategy

\begin{tabular}{cccc}
\hline & Red Agents & Blue Agents & Sum \\
\hline Token $r$ & $n_{r} / 2$ & 0 & $n_{r} / 2$ \\
Token $b$ & 0 & $n_{b} / 2$ & $n_{b} / 2$ \\
Good & $n_{r} / 2$ & $n_{b} / 2$ & $\left(n_{r}+n_{b}\right) / 2$ \\
Sum & $n_{r}$ & $n_{b}$ & $n_{r}+n_{b}$ \\
\hline
\end{tabular}

The expected payoff if following the equilibrium strategy is

$$
r V_{r 0}=\underbrace{\alpha_{r r} \frac{n_{r} / 2}{n_{r}-1}}_{\text {meets a home buyer }}\left(V_{r r}-V_{r 0}\right)
$$

with

$$
r V_{r r}=\underbrace{\alpha_{r r} \frac{n_{r} / 2}{n_{r}-1}}_{\text {meets a home seller }}\left(u+V_{r 0}-V_{r r}\right) .
$$

We can use the two equations above to derive $\left(V_{r 0}, V_{r r}\right)$; denote $V_{r 0}$ in this situation $V_{r 0}$, with "e" for equilibrium strategy.

Deviation Strategy (i)-Accept Both Currencies. If a Red agent follows strategy (i) to accept both tokens, there are two possible asset distribution profiles characterized by $s \in\{0,1\}$, the number of blue tokens held by Red agents.

Table C3: Asset Distribution in Regime $N$ if a Red Agent Accepts Both Currencies

\begin{tabular}{cccc}
\hline & Red Agents & Blue Agents & Sum \\
\hline Token $r$ & $n_{r} / 2$ & 0 & $n_{r} / 2$ \\
Token $b$ & $s \in\{0,1\}$ & $n_{b} / 2-s$ & $n_{b} / 2$ \\
Good & $n_{r} / 2-s$ & $n_{r} / 2+s$ & $n_{r}$ \\
Sum & $n_{r}$ & $n_{b}$ & $n_{r}+n_{b}$ \\
\hline
\end{tabular}

The expected payoff for an individual Red agent who follows deviation strategy (i) is

\footnotetext{
${ }^{1}$ The analysis presented here is for treatments without government agents. Similar analysis can be conducted for the case with government agents.
} 


$$
r V_{r 0}=\underbrace{\alpha_{r r} \frac{n_{r} / 2}{n_{r}-1}}_{\text {meets a home buyer }}\left(V_{r r}-V_{r 0}\right)+\underbrace{\alpha_{r b} \frac{1}{2}}_{\text {meets a foreign buyer }}\left(V_{r b}-V_{r 0}\right)
$$

where

$$
\begin{aligned}
r V_{r r} & =\underbrace{\alpha_{r r} \frac{n_{r} / 2}{n_{r}-1}}_{\text {meets a home seller }}\left(u+V_{r 0}-V_{r r}\right) \\
r V_{r b} & =\underbrace{\alpha_{r b} \frac{n_{b} / 2+1}{n_{b}}}_{\text {meets a foreign seller }}\left(u+V_{r 0}-V_{r b}\right)
\end{aligned}
$$

Note that if the individual Red agent is a seller or holds token $r$, he/she knows that the state of the economy is $s=0$; similarly, if the agent holds $b$, he/she knows the state of the world is $s=1$. We can use the three equations above to derive $\left(V_{r 0}, V_{r r}, V_{r b}\right)$; denote $V_{r 0}$ in this situation $V_{r 0}$, with "i" for deviation strategy (i).

Deviation Strategy (ii)-Only Accept Foreign Currency. If an individual Red agent follows strategy (ii), the possible asset distribution profiles are the same as when the agent follows deviation strategy (i). The expected payoff following strategy (ii) is

$$
r V_{r 0}=\underbrace{\alpha_{r b} \frac{1}{2}}_{\text {meets a foreign buyer }}\left(V_{r b}-V_{r 0}\right)
$$

with

$$
r V_{r b}=\underbrace{\alpha_{r b} \frac{n_{b} / 2+1}{n_{b}}}_{\text {meets a foreign seller }}\left(u+V_{r 0}-V_{r b}\right)
$$

The two equations can be used to solve for $\left(V_{r 0}, V_{r b}\right)$; denote $V_{r 0}$ in this situation $V_{r 0}$, with "ii" for deviation strategy (ii).

Similar to the discussion of strategy (i), here the agent can infer the state of the world by his/her own asset holding: $s=0$ if the agent is a seller, and $s=1$ if the agent is a buyer holding token $b$. This result breaks down when we discuss the case with one single international currency in the next section.

Equilibrium Condition for Red Agents: check $V_{r 0}>V_{r 0}$ and $V_{r 0}>V_{r 0}$.

\section{Regime $R$ and $B$ : One International Currency}

Let us consider the case where token $r$ is accepted by all agents, while token $b$ circulates only nationally. In terms of equilibrium strategies, agents $R$ accept only currency $r$, and agents $B$ accept both currencies.

\subsection{Deviation by a Red Agent}

Possible profitable deviation strategies for a Red agent are (i) accept both currencies (label it R2), and (ii) accept only token $b$ (label it $R b$ ).

Table C4: Red Agent's Equilibrium and Deviation Strategies in Regime $R$

\begin{tabular}{cccc}
\hline & Equilibrium & Deviation $R 2$ & Deviation $R b$ \\
\hline Token $r$ & 1 & 1 & 0 \\
Token $b$ & 0 & 1 & 1 \\
\hline
\end{tabular}

Equilibrium Strategy-Accept Only Home Currency. We will first calculate the expected payoff for a Red agent following the equilibrium strategy. Define $S \equiv \min \left(n_{r} / 2, n_{b}\right)$. If everyone follows the equilibrium strategy, the distribution of assets has $S+1$ possible realizations, with each state, $s \in\{0,1, \ldots, S\}$, being characterized by the number of tokens $r$ held by agents $B$. Let $p_{s}$ be the density of state $s$.

Table C5: Asset Distribution in Regime $R$ If a Red Agent Adopts Deviation Strategy $R 2$

\begin{tabular}{cccc}
\hline & Red Agents & Blue Agents & Sum \\
\hline Token $r$ & $n_{r} / 2-s$ & $s \in\{0,1, \ldots, S\}$ & $n_{r} / 2$ \\
Token $b$ & 0 & $n_{b} / 2$ & $n_{b} / 2$ \\
Good & $n_{r} / 2+s$ & $n_{b} / 2-s$ & $\left(n_{r}+n_{b}\right) / 2$ \\
Sum & $n_{r}$ & $n_{b}$ & $n_{r}+n_{b}$ \\
\hline
\end{tabular}


The agent can be a seller or a buyer holding $r$ in multiple states, and the value function could be different in each of these states. In principle, we need to solve $S+1$ value functions for a seller, and another $S$ for a buyer with $r .^{2}$

The expected payoff if adopting the equilibrium strategy in state $s \in\{0,1, \ldots, S\}$ is

$$
\begin{aligned}
\frac{1}{\beta} V_{r 0, s}= & \underbrace{\alpha_{r r} \frac{n_{r} / 2-s}{n_{r}-1}}_{\text {meets a Red agent with r }} \sum_{s^{\prime}=0}^{S-1} p\left(s^{\prime} \mid s, r r\right) V_{r r, s^{\prime}} \\
& +\underbrace{\alpha_{r b} \frac{s}{n_{b}}}_{\text {meet a Blue agent with r }} \sum_{s^{\prime}=0}^{S-1} p\left(s^{\prime} \mid s, b r\right) V_{r r, s^{\prime}} \\
& +\left(1-\alpha_{r r} \frac{n_{r} / 2-s}{n_{r}-1}-\alpha_{r b} \frac{s}{n_{b}}\right) \sum_{s^{\prime}=0}^{S} p\left(s^{\prime} \mid s, r^{-}\right) V_{r 0, s^{\prime}}
\end{aligned}
$$

where " $\mid s, r r "$ means conditional on being in state $s$ and meeting a Red agent holding $r, " \mid s, b r$ " means conditional on being in state $s$ and meeting a Blue agent holding $r$, and " $s, r^{-"}$ means conditional on being in state $s$ and meeting an agent who does not have $r$. The value function $V_{r r, s}$, with $s \in\{0,1, \ldots, S\}$, satisfies

$$
\begin{aligned}
V_{r r, s}= & \underbrace{\alpha_{r r} \frac{n_{r} / 2+s}{n_{r}-1}}_{\text {meets a Red seller }}\left(u+\beta \sum_{s^{\prime}=0}^{S} p\left(s^{\prime} \mid s, R 0\right) V_{r 0, s^{\prime}}\right) \\
& +\underbrace{\alpha_{r b} \frac{n_{b} / 2-s}{n_{b}}}_{\text {meets a Blue seller }}\left(u+\beta \sum_{s^{\prime}=0}^{S} p\left(s^{\prime} \mid s, b 0\right) V_{r 0, s^{\prime}}\right) \\
& +\left(1-\alpha_{r r} \frac{n_{r} / 2+s}{n_{r}-1}-\alpha_{r b} \frac{n_{b} / 2-s}{n_{b}}\right) \beta \sum_{s^{\prime}=0}^{S-1} p\left(s^{\prime} \mid s, \text { seller }^{-}\right) V_{r r, s^{\prime}} .
\end{aligned}
$$

where "|s, $r 0 "$ ", "|s, b0", and "|s, seller-" mean conditional on being in state $s$ and meeting a Red seller, a Blue seller, or a buyer, respectively.

However, it is reasonable to assume that subjects do not know the state of the world they are in. As a result, we can talk about the value function in expected terms. This simplifies the value functions to

$$
\begin{aligned}
r V_{r 0} & =(\underbrace{\alpha_{r b}^{\frac{\bar{s}}{n_{b}}}}_{\text {meets a Red agent with token r } \quad \underbrace{\alpha_{r r} \frac{n_{r} / 2-\bar{s}}{n_{r}-1}}_{\text {meets a Blue agent with token } r})})\left(V_{r r}-V_{r 0}\right) \\
& =\left(\alpha_{r r} \frac{n_{r}}{n_{r}-1} m_{R r}+\alpha_{r b} m_{B r}\right)\left(V_{r r}-V_{r 0}\right), \\
r V_{r r} & =(\underbrace{\alpha_{r r} \frac{n_{r} / 2+\bar{s}}{n_{r}-1}}_{\text {meets a Red seller }}+\underbrace{\alpha_{r b} \frac{n_{b} / 2-\bar{s}}{n_{b}}}_{\text {meets a Blue seller }})\left(u+V_{r 0}-V_{r r}\right) \\
& =\left(\alpha_{r r} \frac{n_{r}}{n_{r}-1} m_{R 0}+\alpha_{r b} m_{b 0}\right)\left(u+V_{r 0}-V_{r r}\right),
\end{aligned}
$$

where $\bar{s}$ is the expected number of tokens $r$ held by Blue agents. We denote the solution as $V_{r 0}$ and $V_{r r}$. We can then simulate the economy, assuming that everybody plays the equilibrium strategy, to find the expected value of the distribution of assets among subjects $\left\{m_{R r}, m_{r b}, m_{R 0} ; m_{B r}, m_{b b}, m_{b 0}\right\}$, and plug in the $m$ 's to replace $\bar{s}$. Note that we scale the $m$ 's for home meetings by $n_{r} /\left(n_{r}-1\right)$ because the $m$ 's are distributions across the whole population, including the agent under consideration (the scale term approaches 1 when $n_{r}$ increases). ${ }^{3}$

Similarly, we can use the simulated distributions to find value functions if a single Red agent follows a deviation strategy.

\footnotetext{
${ }^{2}$ A buyer holding $r$ knows that $s \in\{0,1, \ldots, S-1\}$.

${ }^{3}$ We are aware that, while calculating $W_{R r}$, the state $s=S$ is not possible because one unit of token $r$ is held by the agent under consideration, and we should adjust for that in principle. However, simulations show that the state $s=S$ is very rare so that the effect of ignoring it is negligible.
} 
Deviation Strategies $R 2$. If the individual Red agent deviates by accepting both tokens $r$ and $b$, then the value functions are (note that we need to simulate the distribution again assuming that the individual agent $r$ follows the deviation strategy and all other subjects follow the equilibrium strategy)

$$
\begin{aligned}
r V_{r 0}= & \left(\alpha_{r r} \frac{n_{r}}{n_{r}-1} m_{R r}+\alpha_{r b} m_{B r}\right)\left(V_{r r}-V_{r 0}\right) \\
& +\alpha_{r b} m_{b b}\left(V_{r b}-V_{r 0}\right) \\
r V_{r r}= & \left(\alpha_{r r} \frac{n_{r}}{n_{r}-1} m_{r 0}+\alpha_{r b} m_{b 0}\right)\left(u+V_{r 0}-V_{r r}\right) \\
r V_{r b}= & \alpha_{r b} m_{b 0}\left(u+V_{r 0}-V_{r b}\right)
\end{aligned}
$$

Denote the solution as $\left(V_{r 0}, V_{r r}, V_{r b}^{R 2}\right)$.

Deviation Strategies $R b$. If the individual agent $r$ deviates by accepting only token $b$, then the value functions are (again recompute the simulated distribution)

$$
\begin{aligned}
& r V_{r 0}=\alpha_{r b} m_{b b}\left(V_{r b}-V_{r 0}\right) \\
& r V_{r b}=\alpha_{r b} m_{b 0}\left(u+V_{r 0}-V_{r b}\right)
\end{aligned}
$$

Denote the solution as $\left(V_{r 0}, V_{r b}^{R b}\right)$.

To ensure Red agents do not deviate from the equilibrium strategy, check $V_{r 0}>V_{r 0}$ and $V_{r 0}>V_{r 0}$.

\subsection{Deviation by a Blue Agent}

We can follow the same steps to analyze the incentive for an individual Blue agent to deviate from the equilibrium strategy. Possible profitable deviation strategies for a Blue agent are (i) accept only home currency $(B b)$, and (ii) accept only foreign currency $(B r)$.

Table C6: Blue Agent's Equilibrium and Deviation Strategies in Regime $R$

\begin{tabular}{cccc}
\hline & Equilibrium & Deviation $B b$ & Deviation $\mathrm{Br}$ \\
\hline Token $r$ & 1 & 0 & 1 \\
Token $b$ & 1 & 1 & 0 \\
\hline
\end{tabular}

Equilibrium Strategy. An individual Blue agent's value functions, if everybody follows the equilibrium strategy, are

$$
\begin{aligned}
r V_{b 0}= & \left(\alpha_{b r} m_{R r}+\alpha_{b b} \frac{n_{b}}{n_{b}-1} m_{B r}\right)\left(V_{B r}-V_{b 0}\right) \\
& +\alpha_{b b} \frac{n_{b}}{n_{b}-1} m_{b b}\left(V_{b b}-V_{b 0}\right) \\
r V_{b r}= & \left(\alpha_{r r} m_{r 0}+\alpha_{b b} \frac{n_{b}}{n_{b}-1} m_{b 0}\right)\left(u+V_{b 0}-V_{b r}\right) \\
r V_{b b}= & \alpha_{b b} \frac{n_{b}}{n_{b}-1} m_{b 0}\left(u+V_{b 0}-V_{b b}\right)
\end{aligned}
$$

Denote the solution as $\left(V_{b 0}^{e}, V_{B r}^{e}, V_{b b}^{e}\right)$.

Deviation strategy $B b$. If the individual Blue agent deviates by accepting only token $b$, the value functions are

$$
\begin{aligned}
r V_{b 0} & =\alpha_{b b} \frac{n_{b}}{n_{b}-1} m_{b b}\left(V_{b b}-V_{b 0}\right) \\
r V_{b b} & =\alpha_{b b} \frac{n_{b}}{n_{b}-1} m_{b 0}\left(u+V_{b 0}-V_{b b}\right)
\end{aligned}
$$

Denote the solution as $\left(V_{b 0}^{B b}, V_{b b}^{B b}\right)$.

Deviation strategy $B r$. If the individual Blue agent deviates by accepting only token $r$, the value functions are

$$
\begin{aligned}
& r V_{b 0}=\left(\alpha_{b r} m_{R r}+\alpha_{b b} \frac{n_{b}}{n_{b}-1} m_{B r}\right)\left(V_{b r}-V_{b 0}\right) \\
& r V_{b r}=\left(\alpha_{r r} m_{r 0}+\alpha_{b b} \frac{n_{b}}{n_{b}-1} m_{b 0}\right)\left(u+V_{b 0}-V_{b r}\right)
\end{aligned}
$$


Denote the solution as $\left(V_{b 0}^{B r}, V_{b r}^{B r}\right)$.

To ensure that Blue agents do not deviate from the equilibrium strategy, check ${ }_{B 0} V_{b 0}^{e}>V_{b 0}^{B b}$ and $V_{b 0}^{e}>V_{b 0}^{B r}$.

\section{Regime $U$ : Two International Currencies}

The regime with two international currencies is always an equilibrium: given that each currency is accepted by everybody else, it is a dominant strategy for an individual agent to accept both as well. 


\section{Appendix D: Robustness Check with Time Dummies and the Effect of Final Periods}

Table D1: Probit Regression with Time Dummies-Home Token Rejection

\begin{tabular}{llccc}
\hline \hline & & \multicolumn{3}{c}{ All Treatments } \\
& & $(1)$ & $(2)$ & $(3)$ \\
& & All & Red & Blue \\
\hline \multirow{3}{*}{ LowInt } & Coef. & 0.476 & -2.194 & 3.178 \\
& Std.Err. & 1.445 & 2.141 & 2.037 \\
& $\mathrm{t}$ & 0.329 & -1.025 & 1.561 \\
\hline \multirow{4}{*}{ AsySize } & Coef. & -0.368 & 0.011 & -1.160 \\
& Std.Err. & 1.458 & 1.816 & 2.825 \\
& $\mathrm{t}$ & -0.253 & 0.006 & -0.411 \\
\hline \multirow{3}{*}{ Govt } & Coef. & 0.997 & -1.022 & 3.518 \\
& Std.Err. & 1.711 & 2.526 & 2.367 \\
& $\mathrm{t}$ & 0.583 & -0.404 & 1.486 \\
\hline \hline
\end{tabular}

Table D2: Probit Regression with Time Dummies-Foreign Token Rejection



Notes. (1) The regression uses data from all four treatments. (2) We divide each session into 15 segments of 10 rounds and create a dummy variable for each of the segment. We include 14 of the 15 time dummies in the regression in place of the linear trend. (3) The regression results on the time dummies are ommitted. 
Table D3: Effect of Final Periods-Home Token Rejection

\begin{tabular}{|c|c|c|c|c|c|c|c|}
\hline & & (1) & & (2) & & (3) & \\
\hline & & All & & Red & & Blue & \\
\hline & Coef. & -0.106 & $* * *$ & -0.109 & $* * *$ & -0.115 & $* * *$ \\
\hline Period & Std.Err. & 0.013 & & 0.016 & & 0.018 & \\
\hline & $\mathrm{t}$ & -8.452 & & -6.800 & & -6.253 & \\
\hline Final10TokenReject & Coef. & 0.062 & & -0.032 & & 0.128 & * \\
\hline & Std.Err. & 0.060 & & 0.084 & & 0.070 & \\
\hline & $\mathrm{t}$ & 1.030 & & -0.377 & & 1.833 & \\
\hline & Coef. & -0.013 & & -2.547 & & 1.835 & \\
\hline LowInt & Std.Err. & 1.514 & & 2.174 & & 1.783 & \\
\hline & $\mathrm{t}$ & -0.009 & & -1.172 & & 1.029 & \\
\hline & Coef. & -1.326 & & -0.251 & & -2.658 & \\
\hline AsySize & Std.Err. & 1.588 & & 1.924 & & 2.609 & \\
\hline & $\mathrm{t}$ & -0.835 & & -0.131 & & -1.019 & \\
\hline & Coef. & -0.461 & & -0.742 & & 0.000 & \\
\hline Govt & Std.Err. & 2.194 & & 3.057 & & 2.552 & \\
\hline & $\mathrm{t}$ & -0.210 & & -0.243 & & 0.000 & \\
\hline No. of Obs. & & 4866 & & 3041 & & 1825 & \\
\hline No. of Groups & & 220 & & 126 & & 94 & \\
\hline & & (1) & & (2) & & (3) & \\
\hline & & All & & Red & & Blue & \\
\hline & Coef. & -0.115 & $* * *$ & -0.115 & $* * *$ & -0.128 & $* * *$ \\
\hline Period & Std.Err. & 0.014 & & 0.017 & & 0.021 & \\
\hline & $\mathrm{t}$ & -8.442 & & -6.852 & & -6.179 & \\
\hline Final20TokenReject & Coef. & 0.118 & & 0.050 & & 0.198 & \\
\hline & Std.Err. & 0.100 & & 0.117 & & 0.143 & \\
\hline & $\mathrm{t}$ & 1.178 & & 0.426 & & 1.383 & \\
\hline & Coef. & -0.095 & & -2.946 & & -3.382 & \\
\hline LowInt & Std.Err. & 1.576 & & 2.272 & & 3.001 & \\
\hline & $\mathrm{t}$ & -0.060 & & -1.297 & & -1.127 & \\
\hline & Coef. & -1.757 & $*$ & -0.882 & $* *$ & -1.670 & \\
\hline AsySize & Std.Err. & 1.743 & & 2.057 & & 5.419 & \\
\hline & $\mathrm{t}$ & -1.008 & & -0.429 & & -0.308 & \\
\hline & Coef. & -1.308 & $* * *$ & -2.329 & $* * *$ & -0.718 & \\
\hline Govt & Std.Err. & 2.517 & & 3.339 & & 3.271 & \\
\hline & $\mathrm{t}$ & -0.520 & & -0.698 & & -0.219 & \\
\hline No. of Obs. & & 4554 & & 2840 & & 1714 & \\
\hline No. of Groups & & 220 & & 126 & & 94 & \\
\hline
\end{tabular}

Notes. (1) The regressions use data from all four treatments. (2) The first (second) table shows the effect of token rejection rate in the last 10 (20) periods. 
Table D4: Effect of Final Periods-Foreign Token Rejection

\begin{tabular}{|c|c|c|c|c|c|c|c|}
\hline & & $\begin{array}{l}(1) \\
\text { All }\end{array}$ & & $\begin{array}{l}(2) \\
\text { Red }\end{array}$ & & $\begin{array}{c}(3) \\
\text { Blue }\end{array}$ & \\
\hline & Coef. & -0.075 & $* * *$ & -0.058 & $* * *$ & -0.088 & $* * *$ \\
\hline \multirow[t]{2}{*}{ Period } & Std.Err. & 0.012 & & 0.015 & & 0.019 & \\
\hline & $\mathrm{t}$ & -6.134 & & -3.807 & & -4.710 & \\
\hline \multirow[t]{4}{*}{ Final10TokenReject } & Coef. & -0.054 & & -0.089 & & -0.042 & \\
\hline & Std.Err. & 0.131 & & 0.175 & & 0.172 & \\
\hline & $\mathrm{t}$ & -0.410 & & -0.511 & & -0.244 & \\
\hline & Coef. & 4.185 & & 2.084 & & 6.183 & \\
\hline \multirow{3}{*}{ LowInt } & Std.Err. & 3.228 & & 4.296 & & 4.252 & \\
\hline & $\mathrm{t}$ & 1.297 & & 0.485 & & 1.454 & \\
\hline & Coef. & 7.377 & $* *$ & 10.363 & $* * *$ & 0.942 & \\
\hline \multirow[t]{3}{*}{ AsySize } & Std.Err. & 3.352 & & 3.982 & & 5.101 & \\
\hline & $\mathrm{t}$ & 2.201 & & 2.602 & & 0.185 & \\
\hline & Coef. & 30.895 & $* * *$ & 32.903 & $* * *$ & 28.518 & $* * *$ \\
\hline \multirow[t]{2}{*}{ Govt } & Std.Err. & 4.361 & & 5.849 & & 5.760 & \\
\hline & $\mathrm{t}$ & 7.085 & & 5.625 & & 4.951 & \\
\hline No. of Obs. & & 3359 & & 1700 & & 1659 & \\
\hline \multirow[t]{4}{*}{ No. of Groups } & & 220 & & 126 & & 94 & \\
\hline & & (1) & & (2) & & (3) & \\
\hline & & All & & Red & & Blue & \\
\hline & Coef. & -0.089 & $* * *$ & -0.074 & $* * *$ & -0.099 & $* * *$ \\
\hline \multirow[t]{2}{*}{ Period } & Std.Err. & 0.014 & & 0.017 & & 0.021 & \\
\hline & $\mathrm{t}$ & -6.363 & & -4.267 & & -4.661 & \\
\hline \multirow[t]{4}{*}{ Final20TokenReject } & $\overline{\text { Coef. }}$ & 0.150 & & -0.001 & & 0.320 & \\
\hline & Std.Err. & 0.217 & & 0.267 & & 0.317 & \\
\hline & $\mathrm{t}$ & 0.692 & & -0.004 & & 1.009 & \\
\hline & Coef. & 3.401 & & 1.079 & & 5.343 & \\
\hline \multirow[t]{3}{*}{ LowInt } & Std.Err. & 3.337 & & 4.411 & & 4.367 & \\
\hline & $\mathrm{t}$ & 1.019 & & 0.245 & & 1.224 & \\
\hline & Coef. & 5.947 & $*$ & 9.747 & $* *$ & -1.670 & \\
\hline \multirow[t]{2}{*}{ AsySize } & Std.Err. & 3.610 & & 4.234 & & 5.419 & \\
\hline & $\mathrm{t}$ & 1.647 & & 2.302 & & -0.308 & \\
\hline \multirow{3}{*}{ Govt } & Coef. & 26.675 & $* * *$ & 30.301 & $* * *$ & 21.992 & $* * *$ \\
\hline & Std.Err. & 4.361 & & 6.749 & & 7.130 & \\
\hline & $\mathrm{t}$ & 5.519 & & 4.490 & & 3.085 & \\
\hline No. of Obs. & & 3104 & & 1566 & & 1538 & \\
\hline No. of Groups & & 220 & & 126 & & 94 & \\
\hline
\end{tabular}

Notes. (1) The regressions use data from all four treatments. (2) The first (second) table shows the effect of token rejection rate in the last 10 (20) periods. 


\section{Appendix E: Experiment Instructions (Govt Treatment) $\underline{\text { Welcome }}$}

You are about to participate in an economics experiment where you will make decisions that could yield a considerable amount of earnings. Your earnings will depend on your own choices and the choices made by others in the experiment. It is therefore important to take your time to understand the instructions.

The earnings you make will be calculated in points. At the end of the experiment, your total points earned will be converted to U.S. Dollars at the rate of 1 point $=\$ 0.02$ (you receive $\$ 2$ for every 100 points you earn). You also receive a \$5 show-up payment regardless of your earnings during the experiment. Your total earnings are paid to you in cash after the experiment ends.

It is important you do not talk or communicate with the others during the experiment. If you have questions or need assistance, please raise your hand and an experimenter will come to you. If you talk, laugh, or exclaim loudly during the session, you may be asked to leave and will not be paid.

This is an experiment in economics decision-making, and is not a psychological experiment with a hidden agenda. That is, we will not be doing anything deceptive and will clarify in these instructions what you will expect during the experiment.

We will first go over the instructions for the experiment and then distribute a short quiz to make sure all participants understand the instructions. After going over the quiz, the experiment will begin. We may distribute a short questionnaire after the experiment ends.

\section{$\underline{\text { Overview }}$}

This experiment consists of 150 rounds. There are 16 participants in today's session: 8 human participants ("traders"), and 8 computerized participants ("robots"). Traders make their own choices and are the individuals you see around you in the laboratory. Robot actions are predetermined by a computer program, which we describe in detail later.

At the beginning of the experiment, all participants are randomly assigned into one of two groups: Group Red or Group Blue. There are 4 traders and 4 robots in each group. All participants will remain in the same group for the entire duration of the experiment.

There are three different objects you may exchange with other participants to earn points: Red tokens, Blue tokens, and Corn. These objects differ in the amount of points they yield. Everyone receives zero points for having either a Red token or a Blue token. However, all participants earn $\underline{10}$ points only by receiving Corn from another participant.

At the beginning of each session, half the participants from Group Red are randomly chosen to receive one unit of Corn. The other half receives one unit of a Red token. Similarly, for Group Blue: half receives Corn, the other half receives a Blue token. 
This means in each round, all participants either have one unit of a Red token, Blue token, or Corn.

\section{Overview of What Happens in a Round}

At the start of each round, you are reminded of the group you are in and the object you currently have (Red token, Blue token, or Corn). There is then a matching process that determines your partner for that round. After being paired, you are given an opportunity to trade goods with your partner. Exchange occurs if and only if you both agree to trade.

You cannot exchange objects with someone holding the same object as you, and you cannot exchange a Red token for a Blue token, or a Blue token for a Red token.

We will now describe in detail the matching process and what happens after a trade.

\section{$\underline{\text { Matching Process }}$}

Each round begins with a matching process that determines your partner for that round.

Your partner is either another trader or a robot, and may either be from Group Red or Group Blue. Remember there are 4 traders and 4 robots in each group. Since there is a new matching process each round, it is likely you will be matched with a different partner in each round.

The table below summarizes the meeting probabilities for each possible occurrence. For instance, meeting probabilities if you are in Group Red are indicated by the second row. There is a $30 \%$ chance you are paired with a trader from Group Red, a 40\% chance with a robot from Group Red, a $15 \%$ chance with a trader from Group Blue, and 15\% chance with a robot from Group Blue.

Similarly, meeting probabilities if you are in Group Blue are indicated by the third row.

These probabilities remain the same throughout the experiment and will appear on your computer screen.

Table: Meeting Probabilities

\begin{tabular}{|c|l|l|l|l|l|l|}
\hline $\begin{array}{c}\text { You, Other } \\
\downarrow\end{array}$ & $\begin{array}{l}\text { Group } \\
\text { Red } \\
\text { Trader }\end{array}$ & $\begin{array}{l}\text { Group } \\
\text { Red } \\
\text { Robot }\end{array}$ & $\begin{array}{l}\text { Group Red } \\
\text { Sum } \\
\text { (Trader+Robot) }\end{array}$ & $\begin{array}{l}\text { Group } \\
\text { Blue } \\
\text { Trader }\end{array}$ & $\begin{array}{l}\text { Group } \\
\text { Blue } \\
\text { Robot }\end{array}$ & $\begin{array}{l}\text { Group Blue } \\
\text { Sum } \\
\text { (Trader+Robot) }\end{array}$ \\
\hline Group Red & $30 \%$ & $40 \%$ & $70 \%$ & $15 \%$ & $15 \%$ & $30 \%$ \\
\hline Group Blue & $15 \%$ & $15 \%$ & $30 \%$ & $30 \%$ & $40 \%$ & $70 \%$ \\
\hline
\end{tabular}

After being matched, you will be told your partner's good for the current round. You will not be given any other information about your partner: i.e., you will not have any information about their group or what they did in previous rounds, and they will not have any information about your group or what you did in previous rounds. It is important to remember that since there is a new matching process in each round, it is likely you will be matched with a different participant in each round.

\section{Trading Opportunity}

Once matched, you are given an opportunity to exchange your good with your partner's good. Specifically, you will be asked privately on your computer screen if you want to trade goods with 
your partner. You can choose either "Yes" or "No.” All exchanges are one-for-one, which means you can only trade one unit of your good for one unit of your partner's good (you may not trade fractions of a good).

You will not see your partner's answer and your partner will not see your answer until after you both have individually and privately chosen an answer. An exchange of goods occurs if and only if both you and your partner agree to trade.

Remember the only way to obtain points will be to obtain Corn from your partner. Since your current partner may be holding an object different from Corn, it may take more than one round to successfully obtain Corn.

\section{Trading Rules for Robots}

All robots follow a preset rule throughout the experiment.

- A robot with Corn always chooses

o "Yes" (to trade) if its partner has a token of the same color as its group affiliation,

o "No" if its partner has a token of different color from its group affiliation.

- If a robot has a token and its partner has Corn, then the robot always chooses "Yes."

For instance, a Group Red robot with Corn chooses "Yes" if its partner has a Red token and "No" if its partner has a Blue token. If it holds a Red token and its partner has Corn, then it always chooses "Yes."

\section{Outcome of a Round}

If both you and your partner agree to trade, then one of the following will occur:

- If you receive either a Red token or Blue token from your partner, you will earn 0 points.

- If you receive Corn from your partner, you will earn 10 points.

In the next round, you will have your partner's good, and your partner will have your good.

If either you or your partner does not agree to trade, then no trade occurs. In that case, neither you nor your partner earns any points.

In the next round, you will continue with the same object you started the round with, and your partner will continue with the same object he or she started the round with.

\section{Figures}

The timing of events in a round is summarized in the top figure, and a screenshot of the experiment is in the bottom figure. 



When a session ends, a message will appear on your screen. You will see your point total for the session. An experimenter will then come around to your station to record your point total and distribute your cash earnings. 


\section{$\underline{\text { Quiz }}$}

Please answer the following questions about today's experiment. You may refer back to the instructions to help choose an answer.

1. Among the following options, which one gives you the most points, if both you and your partner agree to trade?

(a) You trade Corn for your partner's Red token.

(b) You trade Blue token for your partner's Corn.

(c) You trade Corn for your partner's Corn.

2. Please fill in the following table regarding the possible trades according to the instructions with either a "Yes" or "No."

\begin{tabular}{|l|l|l|}
\hline Your Good & Partner's Good & Possible to Trade? \\
\hline Red Token & Red Token & No \\
\hline Blue Token & Blue Token & \\
\hline Corn & Corn & \\
\hline Red Token & Blue Token & \\
\hline Blue Token & Red Token & \\
\hline Red Token & Corn & \\
\hline Corn & Red Token & Yes \\
\hline Blue Token & Corn & \\
\hline Corn & Blue Token & \\
\hline
\end{tabular}

3. Suppose you initially hold a Red token and successfully trade for Corn from your partner. Which of the following are possible in the next round?

(a) You will hold Corn in the next round.

(b) You may be matched with a new partner.

(c) The session ends.

(d) All of the above are possible.

4. Before deciding whether to trade, will you know the ID or group of your partner?

5. Please fill in the following table regarding the choices made by robots according to the instructions with either a "Yes" or "No."

\begin{tabular}{|l|l|l|l|}
\hline \multirow{4}{*}{ Own Good } & Partner's Good & $\begin{array}{l}\text { Possible to } \\
\text { Trade? }\end{array}$ \\
\hline \multirow{3}{*}{ Group Red robot } & Corn & Red token & Yes \\
\hline & Corn & Blue token & \\
\hline Red token & Corn & \\
\cline { 2 - 4 } Group Blue robot & Corn & Blue token & \\
\hline & Corn & Red token & \\
\hline & Red token & Corn & Yes \\
\hline
\end{tabular}

